\title{
The chemiluminescent peroxyoxalate system: state of the art almost 50 years from its discovery
}

\author{
Luiz Francisco M. L. Ciscato, ${ }^{\text {a,c* }}$ Felipe A. Augusto, ${ }^{a}$ Dieter Weiss, ${ }^{\text {b }}$ Fernando H. \\ Bartoloni, ${ }^{\mathrm{c}}$ Steffen Albrecht ${ }^{\mathrm{d}}$, Herbert Brandl, ${ }^{\mathrm{e}}$ Thomas Zimmermann, ${ }^{\mathrm{d}}$ \\ and Wilhelm J. Baader ${ }^{\mathrm{a}}$ \\ ${ }^{a}$ Departamento de Química Fundamental, Instituto de Química da Universidade de São Paulo, \\ Av. Prof. Lineu Prestes, 748, Sala 1257. São Paulo, SP, 05508-900, Brazil \\ ${ }^{b}$ Institut für Organische Chemie und Makromolekulare Chemie, Friedrich-Schiller Universität, \\ Humboldtstrasse 10, Jena, D-07743, Germany \\ ${ }^{c}$ Centro de Ciências Naturais e Humanas, Universidade Federal do ABC, Rua Santa Adélia, 166. \\ Santo André, SP, 09210-170, Brazil \\ ${ }^{d}$ Klinik und Poliklinik für Frauenheilkunde und Geburtshilfe, Technische Universität Dresden, \\ Fetscherstrasse 74, Dresden, D-01307, Germany. \\ ${ }^{e}$ Gymnasium in Kaltenkirchen, Flottkamp 34, Kaltenkirchen, D-24568, Germany \\ E-mail: luiz.ciscato@ufabc.edu.br
}

This article is dedicated to Prof. Dr. Rainer Beckert on the occasion of his $60^{\text {th }}$ birthday

\begin{abstract}
Almost fifty years after the discovery of the peroxyoxalate reaction by E. A. Chandross in the early nineteen sixties, this review article intends to give a general overview on mechanistic aspects of this system and to describe the principles of its analytical application. After a short general introduction on the principles of chemiluminescence and the history of peroxyoxalate discovery, mechanistic aspects of high-energy intermediate formation, its structure and its reaction with an activator in the peroxyoxalate system are discussed. Finally, analytical applications of peroxyoxalate chemiluminescence are exemplified using representative recent examples, including oxalic acid detection in biological samples.
\end{abstract}

Keywords: Peroxyoxalate, chemiluminescence, organic peroxides, excited states

\section{Table of Contents}

1. Introduction

1.1. Principles of chemiluminescence 
1.2. Discovery of the peroxyoxalate reaction

2. Formation of the high-energy intermediate

3. Structure of the high-energy intermediate

4. Chemiexcitation mechanism

5. Analytical applications of peroxyoxalate chemiluminescence

6. Conclusions

7. Acknowledgements

8. References

9. Authors' biographies

\section{Introduction}

\subsection{Principles of chemiluminescence}

Chemiluminescence refers to a process in which light emission is obtained as the product of a chemical transformation. The related process of bioluminescence - emission of light by living organisms - was described by Aristotle already in ancient times. ${ }^{1}$ The physician Hennig Brandt in 1669 , by reducing the solid residues from urine distillation, produced a material which showed the outstanding property of glowing in the dark with a bluish light emission, without the need of prior exposure to light, heating or mechanical treatment. This discovery of light emission by chemical substances was then called miraculous light ("phosphorous mirabilis") and the phenomenon is now a well-known property of phosphorous exposed to ambient oxygen. ${ }^{1}$ Thereafter, chemiluminescence has been described by Wiedemann in 1888, who also forwarded the first definition of the process: "Das bei chemischen Prozessen auftretende Leuchten würde Chemilumineszenz genannt", which, translated from German, means "The shining occurring during chemical processes should be called chemiluminescence". ${ }^{2}$ Wiedemann proposed this classification after analysis of a work from Radziszewski published almost a decade before, in which he described the light emission occurring during autooxidation of lophine (2,4,5triphenylimidazole, 1). ${ }^{3}$ Thereafter, other chemiluminescence systems were reported, like the transition metals catalyzed oxidation of luminol (5-amino-2,3-dihydro-1,4-phtalazinedione, 2) by $\mathrm{H}_{2} \mathrm{O}_{2}$, described by Albrecht in $1928,{ }^{4}$ and the oxidation of lucigenin ( $N, N^{\prime}$-dimethyl-9,9'bisacridinium salt, 3) described by Gleu and Petsch. ${ }^{5}$

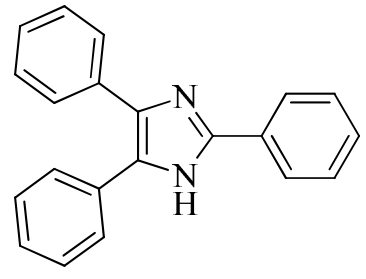

1<smiles>Nc1cccc2c(=O)[nH][nH]c(=O)c12</smiles>

2<smiles>C[n+]1c2ccccc2c(-c2c3ccccc3[n+](C)c3ccccc23)c2ccccc21</smiles>

3 
In a general way, any chemiluminescent transformation involves the formation of electronically excited states from an exothermic reaction, in one or more steps. Obviously not every exothermic reaction leads to formation of excited states, because in a conventional thermal reaction the energy is used to form or cleave bonds between the atomic centers involved; the energy is utilized to promote the reagent to the transition state level which forms the product in a vibrationally excited state, which decays to the vibrational ground state with emission of thermal energy in the form of heat. ${ }^{6}$ A chemiluminescent reaction has to be necessarily highly exothermic; however, it may be chemiluminescent only when the transition state possesses sufficient energy to form one of the products in its electronically excited state (Figure 1).

(a)

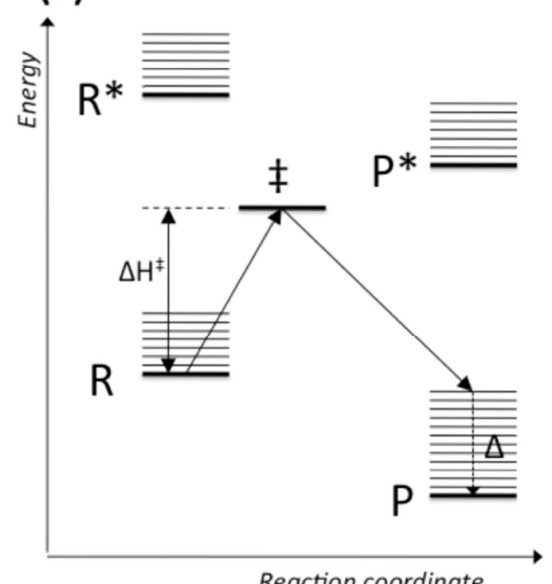

Reaction coordinate (b)

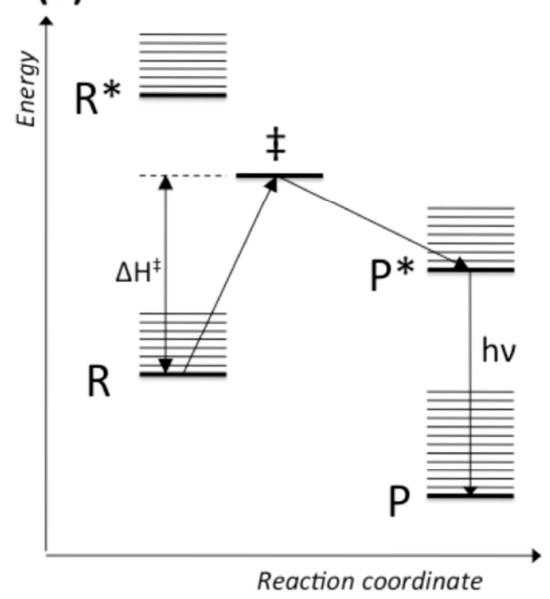

Figure 1. Potential energy diagrams for (a) an exothermic reaction $R \rightarrow P$ and (b) a chemiluminescent reaction $\mathrm{R} \rightarrow \mathrm{P}^{*}$.

Since the energy released during the chemiexcitation step has to be high enough to produce electronically excited states, reaction exothermicity has to be from 50 to $100 \mathrm{kcal} \mathrm{mol}^{-1}$, which is the excitation energy of most organic chromophores. Also very important is the fact that the excited state of the emitter has to be accessible, making carbonyl compounds and aromatic hydrocarbons suitable substances to transform the chemical energy into electronically excitation energy. ${ }^{7}$ However, even if the energy is sufficient for the occurrence of chemiexcitation, geometric factors have also to be considered. Those factors are normally related to the FranckCondon principle, which states that the time scale of electronic transitions $\left(10^{-16}\right.$ to $\left.10^{-14} \mathrm{~s}\right)$ is smaller than the time scale of nuclear movements $\left(10^{-13}\right.$ to $\left.10^{-12} \mathrm{~s}\right)$, and therefore, electronic transitions take place much faster than the rearrangement of atoms in the molecular structure. ${ }^{8} \mathrm{~A}$ chemical reaction, in which the structure of the transition state is similar to the structure of the product's excited state, and dissimilar to the structure of the product's ground state, has a greater probability of forming excited states. ${ }^{9}$ 
The efficiency of a chemiluminescent transformation is defined as the ratio between emitted photons and amount of limiting reagent molecules which reacted. This represents the value of the chemiluminescence quantum yield $\left(\Phi_{\mathrm{CL}}\right)$. In a general way, such a parameter comprises the socalled chemiexcitation quantum yield $\left(\Phi_{\mathrm{ex}}\right)$ which corresponds to the amount of excited state molecules formed in respect to the number of limiting reagent molecules transformed, as well as the emission quantum yield of the excited species (Equation 1), generally its fluorescence quantum yield $\left(\Phi_{\mathrm{Fl}}\right)$.

$$
\Phi_{\mathrm{CL}}=\Phi_{\mathrm{ex}} \Phi_{\mathrm{Fl}}
$$

The process of excited state formation can be better rationalized with the help of the Marcus Theory for electron-transfer reactions, where the energetic and geometric requirements can be evaluated by using potential energy reaction coordinate diagrams. ${ }^{10}$ In non-chemiluminescent exothermic reactions, the activation energy for the formation of the product $\mathrm{P}$ in the electronic ground state $\left(\Delta \mathrm{H}^{\ddagger}\right)$ is lower than the activation energy $\Delta \mathrm{H}^{\ddagger^{*}}$, for the formation of the product in its electronically excited state $\left(\mathrm{P}^{*}\right)$, thus the formation of $\mathrm{P}$ in its ground state is kinetically favorable. This is especially true if the geometry (reaction coordinate position) of the reactants and the ground state products is similar (Figure 2a). Nevertheless, if the geometry of the excited state products is similar to that of the reactants, a chemiluminescent reaction can occur, where $\Delta \mathrm{H}^{\ddagger^{*}}$ is smaller than $\Delta \mathrm{H}^{\ddagger}$, making the excited state of the product more attainable kinetically, consequently, efficient formation of electronically excited states might be observed (Figure 2b). In these cases, a highly exothermic reaction, leading to the formation of products in the ground state, can be kinetically less favorable than the corresponding reaction leading to formation of electronically excited states, due to the fact that the structure of the transition state is more similar to the structure of excited state products than of the ground state products (Figure 2).

(a)

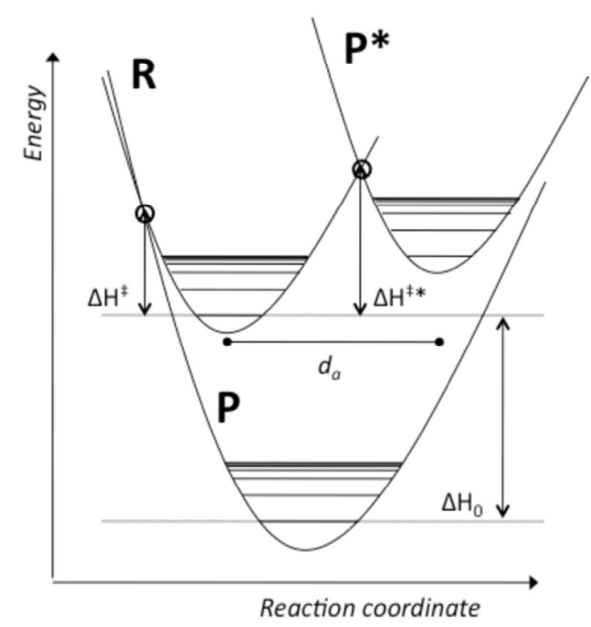

(b)

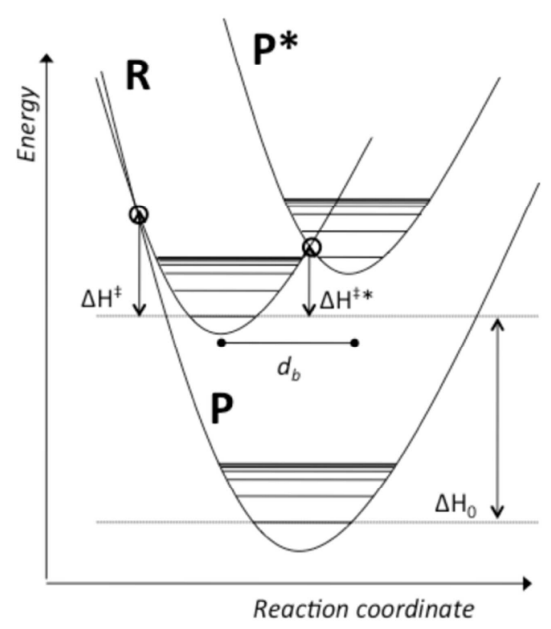

Figure 2. Diagram of reaction coordinates for (a) a non-chemiluminescent exothermic reaction and (b) a chemiluminescent exothermic reaction, according to the Marcus Theory. The distances 
between the equilibrium geometries of $R$ and $P^{*}$ for (a) and (b) are represented as $d_{a}$ and $d_{b}$, respectively.

Chemiluminescence reactions can be generally divided in three main steps: (i) formation of a high-energy intermediate (HEI), in one or more chemical transformations of ground state molecules; (ii) unimolecular decomposition of the HEI or its interaction with other reagents, leading to electronically excited state formation (chemiexcitation step); (iii) decay of this excited state to the ground state accompanied by fluorescence or phosphorescence emission, depending on the multiplicity of the excited state..$^{9,11}$

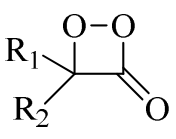

4

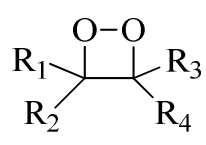

5

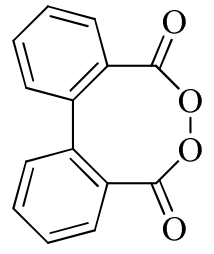

6

With the synthesis of cyclic organic peroxides like 1,2-dioxetanones (4) and 1,2-dioxetanes (5), which are nothing more than isolated HEI, and detailed studies on their chemiluminescent decomposition, two distinct general chemiexcitation mechanisms could be outlined: (i) unimolecular cleavage or rearrangement of molecules with high energy content forming excited states, as in the unimolecular thermal decomposition of 1,2 -dioxetanes; ${ }^{12}$ or (ii) catalyzed decomposition of the high energy peroxide by a suitable activator (ACT), forming the excited state of the ACT, a mechanism known as CIEEL (Chemically Initiated Electron Exchange Luminescence), initially proposed by $\operatorname{Schuster}^{13}$ (Scheme 1). Studies on the chemiluminescence properties of 1,2-dioxetanones ${ }^{14}$ and diphenoyl peroxide ${ }^{15}$ (6) revealed that the observed light emission rate constants $\left(\mathrm{k}_{\mathrm{obs}}\right)$, as well as the chemiluminescence quantum yields $\left(\Phi_{\mathrm{CL}}\right)$, increased proportionally with the concentration of added ACT. Furthermore, a dependency of the $\mathrm{k}_{\mathrm{obs}}$ and the emission intensities with the oxidation potential of the added ACT could be taken as a clearcut evidence for the occurrence of an electron transfer from the ACT to the cyclic peroxide in the rate-limiting step. ${ }^{9,11,13-15}$

On the basis of these and other experimental observations, Schuster and coworkers postulated the CIEEL mechanism, where the first step comprises formation of a charge-transfer type encounter complex $\left(\mathrm{K}_{\mathrm{CT}}\right)$ between the peroxide and the ACT, inside the solvent cavity (Scheme 1). ${ }^{7,13-15}$ Elongation of the relatively weak O-O bond by thermal activation within the charge-transfer complex results in the lowering of the antibonding orbital energy $\left(\sigma^{*}\right)$, permitting the occurrence of an endothermic electron transfer from the ACT to the peroxide $\left(\mathrm{k}_{\mathrm{ET}}\right)$, which is accompanied by the cleavage of the $\mathrm{O}-\mathrm{O}$ bond. Although not being energetically favorable, the electron transfer is possible, as the $\mathrm{O}-\mathrm{O}$ bond cleavage occurs almost simultaneously, making the whole process essentially irreversible (Scheme 1). ${ }^{7,11}$ Subsequently, the radical anion of the 1,2- 
dioxetanone, still inside the solvent cavity with the ACT, undergoes $\mathrm{C}-\mathrm{C}$ bond cleavage $\left(\mathrm{k}_{\text {CLEAv }}\right.$ ), resulting in a neutral compound and a carbonyl radical anion. The new pair of radical ions, the carbonyl radical anion and the radical cation of the ACT, yet inside the solvent cavity, can now undergo an electron back-transfer $\left(\mathrm{k}_{\mathrm{EBT}}\right){ }^{\dagger}$ which can lead to the formation of a singlet excited state $\left(\mathrm{S}_{1}\right)$ of the ACT. Finally, the ACT returns to its ground state with the emission of fluorescence (Scheme 1). Escape of the radical pairs from the solvent cavity in any stage of the reaction will lead to the formation of only ground state products, due to the extremely low probability of radical ion reencounter. ${ }^{7,9,11,13}$

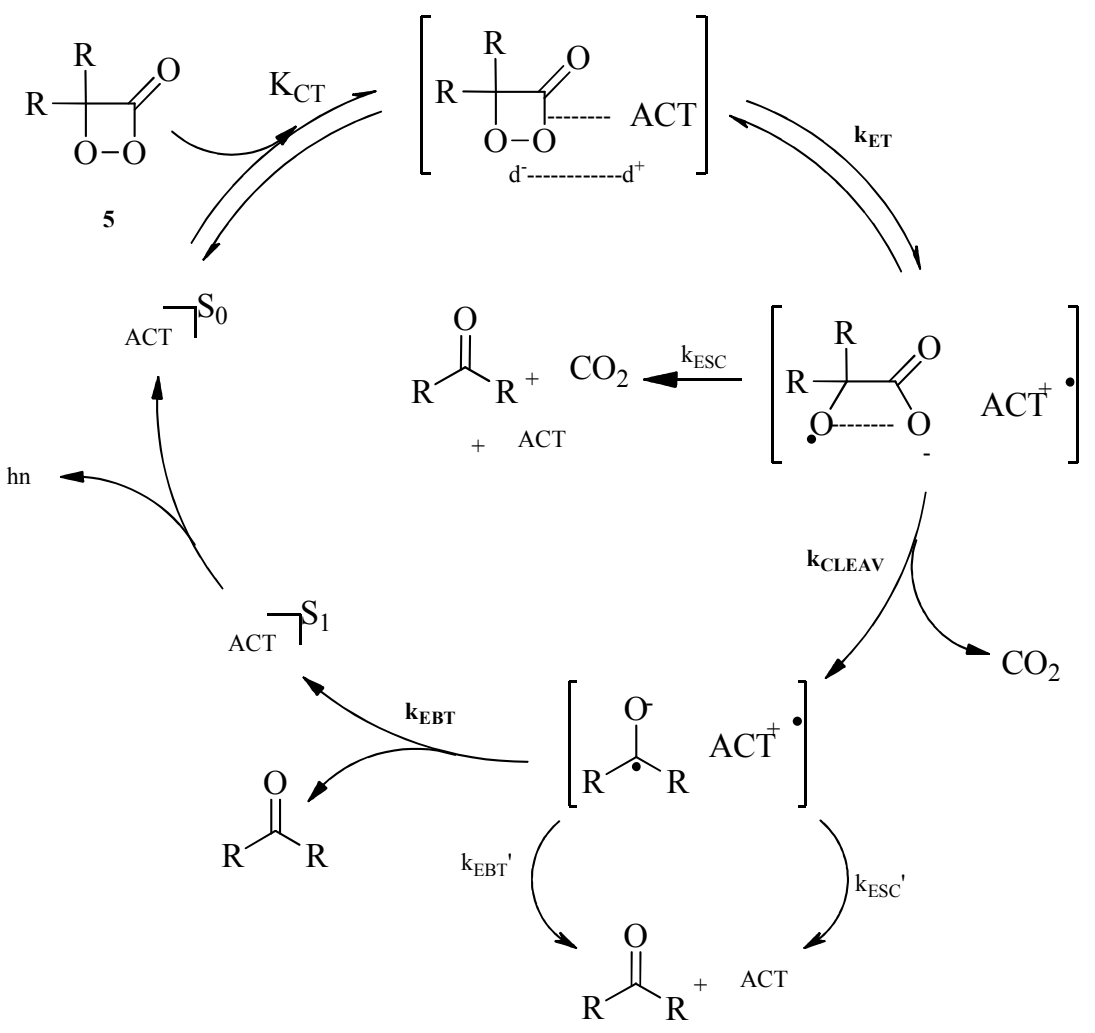

Scheme 1. CIEEL sequence for chemiexcitation of an activator (ACT) by its interaction with a 1,2-dioxetanone (4).

The CIEEL mechanism has been utilized to explain the catalyzed decomposition of several cyclic and linear peroxides, including diphenoyl peroxide, peroxyesters and 1,2-dioxetanones. Special interest has been attained to this mechanism when it was utilized to explain efficient excited state formation in the chemiexcitation step of the firefly luciferin/luciferase

${ }^{\dagger}$ Although the inverse transfer of an electron from the carbonyl radical anion to the activator's radical cation is normally named "back-electron transfer (BET)" in the former literature, we accepted the suggestion of a referee and call this process an "electron back-transfer (EBT)" which appears to be the more reasonable expression. 
bioluminescence. ${ }^{16}$ However, doubts were raised more recently on the validity of this mechanistic scheme, due to divergences about the chemiluminescence quantum yields measured in CIEEL systems. The quantum yield for the diphenoyl peroxide / perylene system was shown to be three orders of magnitude lower than the value initially determined, ${ }^{17,18}$ and since this is the prototype system for the CIEEL proposal, the validity of this mechanism was itself questioned. $^{9,11}$

Contrarily, 1,2-dioxetanes containing substituents with low oxidation potentials, such as [aryl-O]', [aryl-RN] functionalities, decompose apparently via an intramolecular version of the CIEEL mechanism, leading to the efficient generation of singlet-excited carbonyl compounds. Spiroadamantane-substituted 1,2-dioxetanes with a protected phenolate ion substituent are the most studied intramolecular CIEEL systems as these 1,2-dioxetanes proved to be thermally persistent and their decomposition can be achieved on treatment with an appropriate reagent (trigger) to induce phenolate ion release. ${ }^{19}$ These phenolate-initiated intramolecular CIEEL processes provide the basis for numerous commercial applications, most prominently in chemiluminescence immunoassays. ${ }^{20}$ Mechanistic studies from several research groups revealed details of these transformations, which, after deprotection of the phenolate, are believed to be initiated by an intramolecular electron transfer from the phenolate oxygen to the peroxidic bond, analogous to the intermolecular CIEEL mechanism. Cleavage of the central $\mathrm{C}-\mathrm{C}$ bond may occur in two different ways (Scheme 2, pathways A and B) and excited state formation will consequently be due to an intra or intermolecular electron back-transfer. ${ }^{21,22}$ A clear-cut experimental evidence on the occurrence of the initial intramolecular electron transfer has been obtained recently in a mechanistic study on the decomposition of acridinium-substituted 1,2dioxetanes. ${ }^{23}$ However, there is still a controversy with respect to the inter or intramolecular nature of the electron back-transfer (Scheme 2). ${ }^{9,11,21,22}$

\subsection{Discovery of the peroxyoxalate reaction}

The only chemiluminescent system apparently operating by an intermolecular CIEEL mechanism with proved high efficiency is the peroxyoxalate system (PO), capable of producing light emission in yields up to $30 \%$, a value comparable only to enzymatic chemiluminescence systems. ${ }^{11}$ Despite the fact that the PO reaction was discovered - or better to say observed almost fifty years ago, the mechanism of formation and the structure of the high energy intermediate (HEI) and the chemiexcitation pathway are still matter of discussion in the scientific community. ${ }^{9,11}$

The first person to observe and describe the PO system was Edwin A. Chandross, in an article published in Tetrahedron Letters in $1963 .{ }^{24}$ While working as researcher in the Bell Labs, he reported a flash of light coming from the flask where he had just mixed oxalyl chloride, hydrogen peroxide and 9,10-diphenylanthracene (Scheme 3). 


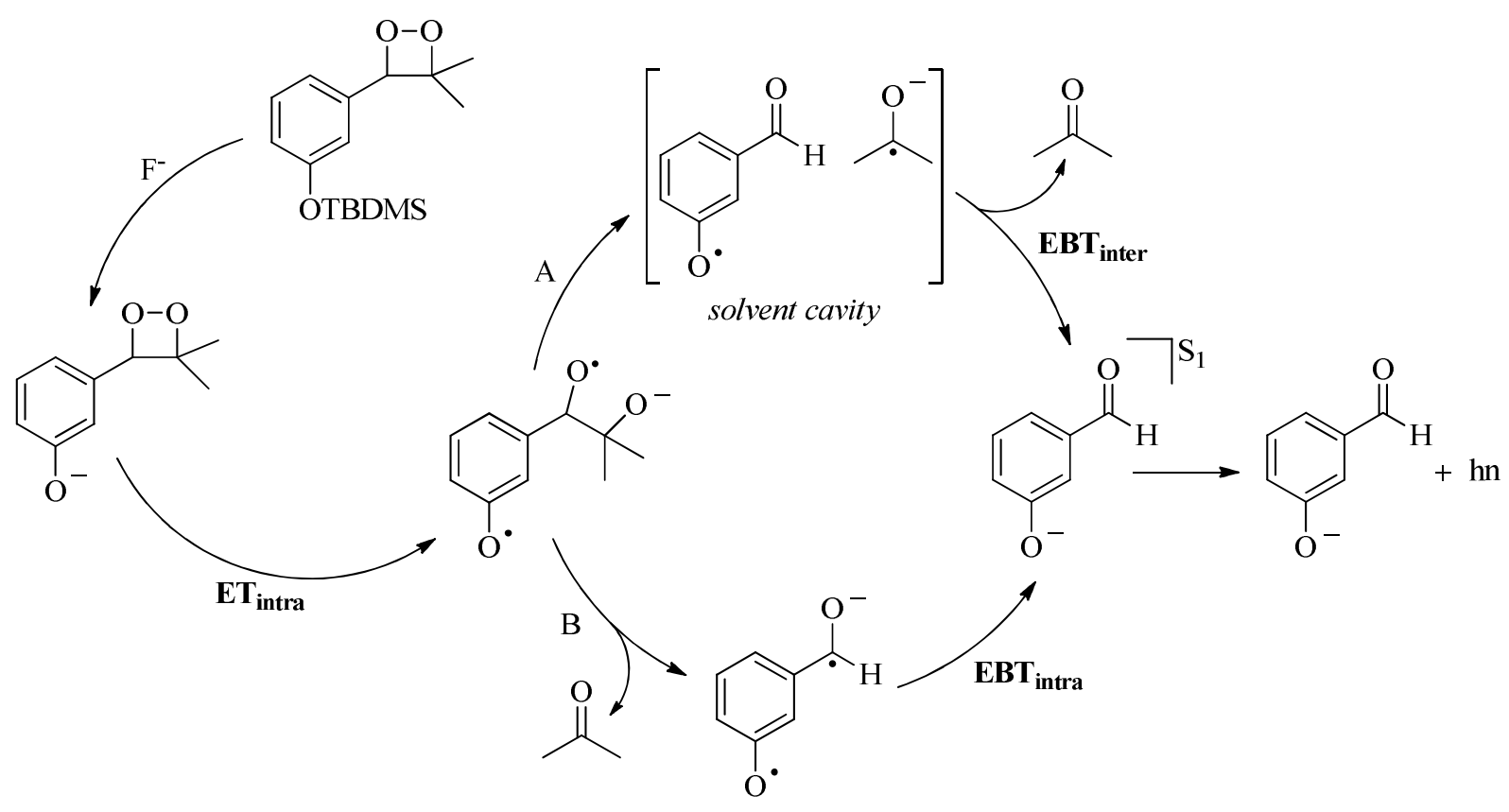

Scheme 2. Mechanism of the induced decomposition of phenolate-substituted 1,2-dioxetanes.

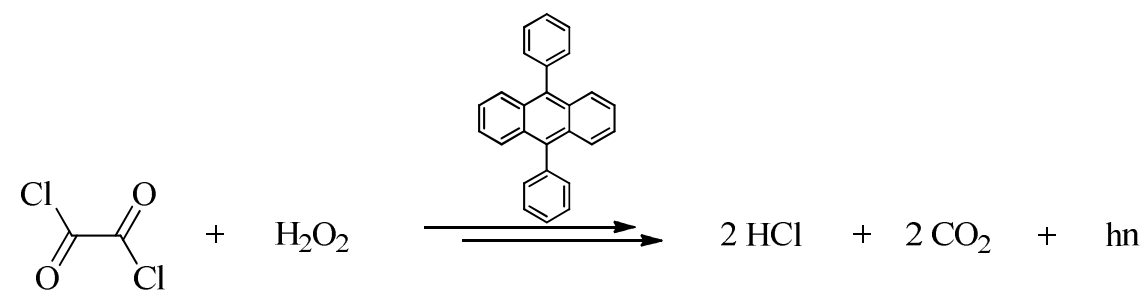

Scheme 3. Chemiluminescent reaction of oxalyl chloride and hydrogen peroxide in the presence of 9,10-diphenylanthracene, as first observed by Chandross. ${ }^{24,25}$

Around 30 years after this discovery, in a book chapter, ${ }^{25}$ Chandross described in detail what he was trying to achieve when he made the famous "peroxyoxalate cocktail". He and his team at the AT\&T Bell Labs in Murray Hill, New Jersey, USA, were looking for evidences for the involvement of the recombination of radical cation and anion pairs in excited state formation; in his words "struggling to find a common thread that could explain a variety of chemiluminescent systems in the literature". One approach to determine the mechanism of chemiluminescent reactions was to study highly exothermic intramolecular decomposition reactions which liberate enough energy to populate an excited state. In this context, a study on the decomposition of the geminal hydroperoxide derived from xanthone appeared to be a good starting point. This diperoxide could be easily synthesized in two steps, first by the reaction of xanthone and oxalyl chloride, forming the geminal dichloride, followed by reaction of this dichloride with hydrogen 
peroxide. The researchers could easily see light emission from the reaction of the crude, unpurified, dichloride with $30 \%$ hydrogen peroxide, but no light emission at all from the reaction of the purified product with the same hydrogen peroxide. This observation quickly led to the investigation of the reaction of oxalyl chloride and hydrogen peroxide as a source of light emission. It was shown indeed that this reaction could excite any fluorescent substance present in the mixture, producing light emission with a wavelength matching the fluorescent emission of the substance. The peroxyoxalate system was discovered. ${ }^{25}$

Already in his 1963 letter, Chandross not only described the PO reaction, but also made a series of experiments trying to gain insight into the nature of the chemiluminescence process. He described that the vapors produced by the reaction had the property of inducing the fluorescence of suitable substances impregnated in filter papers, and suggested the formation of metastable excited electronic state or other highly energetic species. He also observed that the color of the chemiluminescence emission always matched the fluorescence spectrum of the substance added to the reaction mixture. The chromatographic analysis of the gases produced by the reaction showed the formation of considerable amounts of carbon dioxide, carbon monoxide and surprisingly also oxygen, maybe due to decomposition of peroxide intermediates. From known thermodynamic data, Chandross estimated the energy released in the overall reaction as being about $70 \mathrm{kcal} \mathrm{mol}^{-1}$, too low to excite $\mathrm{HCl}, \mathrm{CO}$ and $\mathrm{CO}_{2}$. Therefore, any mechanism able to explain the light emission should involve other species than these final reaction products. ${ }^{25}$

In this first letter, Chandross did not reached any conclusion about the mechanism of this new chemiluminescent system, however he properly suggested the direction of future studies: initially, he proposed that the mechanism has to involve an unknown compound derived from oxalyl chloride and hydrogen peroxide as intermediate, which he named as a high-energy intermediate (HEI); additionally, he pointed out the importance of the "energy transfer" from this HEI to a suitable fluorophore, since this transformation might involve a new mechanistic principle. Those suggestions indicated the main challenges awaiting the researchers willing to study the mechanism of this interesting system.

\section{Formation of the high-energy intermediate}

Since its discovery by Chandross, ${ }^{24}$ the mechanism of the reaction sequence leading to the formation of a high-energy intermediate (HEI) or intermediates of the PO system has been extensively studied. ${ }^{26,27}$ This peroxyoxalate reaction sequence is composed of a great variety of parallel and subsequent steps and its rate depends on both the structure and concentration of substrate and catalyst, as well as the hydrogen peroxide concentration. However, the nature and concentration of the activator (ACT) utilized does not affect the reaction rates, since the chemiexcitation step, consisting in the interaction of the HEI with the ACT, is not ratedetermining. ${ }^{11}$ 
Following the discovery of the PO system by Chandross, Rauhut and coworkers examined the kinetic behavior of the light emission and the total quantum yields for the PO system with oxalyl chloride and hydrogen peroxide in diethyl ether. ${ }^{28}$ The chemiluminescence quantum yields were shown to increase linearly with the hydrogen peroxide concentration, whereas the rate of light emission remained constant in the concentration range employed (1 to $10 \mathrm{mmol} \mathrm{L}^{-1}$ ). However, in the presence of small amounts of water, the reaction rate showed a linear dependence with the hydrogen peroxide concentration. ${ }^{28}$

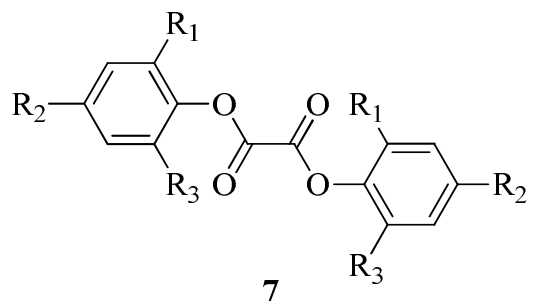

7

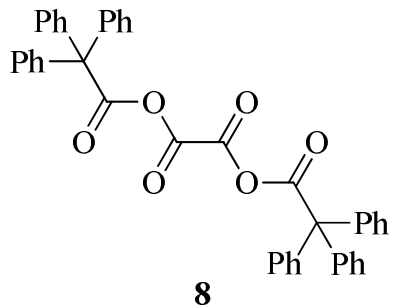

8

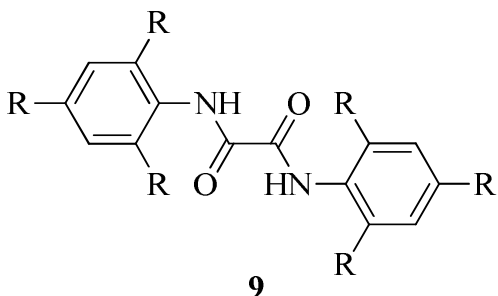

9

As an alternative to the PO system with oxalyl chloride, Rauhut and coworkers reported the reaction of oxalic anhydrides $(\mathbf{8}),{ }^{29}$ aryl oxalates $(7)^{30}$ and oxamides $(\mathbf{9})^{31}$ with hydrogen peroxide, showing maximum quantum yields of $0.13,0.23$ and $0.01 \mathrm{E} \mathrm{mol}^{-1}$ respectively, as compared to a yield of $0.05 \mathrm{E} \mathrm{mol}^{-1}$ obtained with oxalyl chloride. ${ }^{28}$ The high chemiluminescence quantum yields for oxalic esters 7, containing electron withdrawing substituents, made these compounds to be standard reagents for the PO system, also due to the facility of their preparation and their higher stability towards hydrolysis in a comparison to oxalyl chloride.

In a systematic investigation on the PO system with oxalic esters, Rauhut and coworkers studied twenty different symmetrical oxalic esters substituted with functionalized phenyl groups, observing the light emission produced by those esters when they were mixed with hydrogen peroxide in dimethoxyethane (DME) as solvent and 9,10-diphenylanthracene (DPA) as activator, in both basic and acid medium. ${ }^{30}$ The authors did not observe appreciable light emission with any ester in acidic medium, however in basic medium they reported moderate to strong emissions in case of aryl esters substituted by electron-withdrawing groups. The rate of light emission showed to be dependent on the electronegativity of the aryl substituent, however observed rate constants could not be determined due to complex kinetic behavior. ${ }^{30}$

The chemiluminescence quantum yields for the PO system varied at least three orders of magnitude, being the highest for bis(2,4-dinitrophenyl) oxalate (DNPO, $7 \mathrm{R}_{1}=\mathrm{R}_{2}=\mathrm{NO}_{2}, \mathrm{R}_{3}=\mathrm{H}$ ), bis(pentachlorophenyl) oxalate (PCPO) and bis(pentafluorophenyl) oxalate (PFPO). The authors also reported the effect of the peroxide concentration and its structure on the chemiluminescence quantum yields with DNPO as reagent. Using organic peroxides instead of hydrogen peroxide, very low quantum yields were obtained, for example $1 \times 10^{-4} \mathrm{E} \mathrm{mol}^{-1}$ for $t$-butyl hydroperoxide and $1 \times 10^{-5} \mathrm{E} \mathrm{mol}^{-1}$ for benzoyl peroxide. These results emphasize the importance of hydrogen peroxide as oxidant, which provides chemiluminescence quantum yields of $1.7 \times 10^{-1} \mathrm{E} \mathrm{mol}^{-1}$ 
under similar conditions. The authors also tried to correlate the rate of light emission with the rate of reagent disappearance, by monitoring infrared absorptions assigned to the ester carbonyl band at $1806 \mathrm{~cm}^{-1}$. Although no systematic study was performed, the authors reported that the ester consumption was faster than the light emission decay kinetics, indicating the formation of a metastable intermediate. ${ }^{30}$

A complete kinetic study of the reaction of PCPO with hydrogen peroxide and DPA in ethyl acetate and chlorobenzene as solvents was conducted by Catherall and Palmer. ${ }^{32}$ Preliminary investigations revealed that the results could not be reproduced while carrying out the experiments without base as catalyst. Therefore the authors determined the observed rate constants for the emission intensity decay using different concentrations of sodium salicylate as base catalyst. The pseudo-first-order rate constants obtained showed to be independent of the DPA concentration, and reached a maximum of $3.3 \times 10^{-2} \mathrm{~s}^{-1}$ with a salicylate concentration of $2.3 \times 10^{-5} \mathrm{~mol} \mathrm{~L}^{-1}\left([\mathrm{PCPO}]=5 \times 10^{-4} \mathrm{~mol} \mathrm{~L}^{-1},\left[\mathrm{H}_{2} \mathrm{O}_{2}\right]=2 \times 10^{-2} \mathrm{~mol} \mathrm{~L}^{-1},[\mathrm{DPA}]=1 \times 10^{-3} \mathrm{~mol} \mathrm{~L}^{-}\right.$ ${ }^{1}$ ), having reduced values for lower or higher catalyst concentrations. Additionally, the chemiluminescence quantum yields proved to be inversely proportional to the salicylate concentration, while the highest values could be reached in the absence of this reagent. The chemiluminescence decay rate constants were shown to increase with increasing concentration of hydrogen peroxide. The pseudo-first-order decay rate constants showed linear dependence with the hydrogen peroxide concentration with a slope of $0.9 \pm 0.1 \mathrm{~L} \mathrm{~mol}^{-1} \mathrm{~s}^{-1}$ and intercept zero. However, the chemiluminescence quantum yields were reduced with increasing hydrogen peroxide concentrations. $^{32}$

In order to obtain more information about the mechanism of the initial steps of the PO reaction, Orosz ${ }^{33}$ monitored by means of gas chromatography the liberation of substituted phenols in the course of the reaction between several oxalic esters and hydrogen peroxide. Kinetic measurements on the hydrolysis of symmetrical oxalic esters led to the conclusion, that the first molar equivalent of phenol was produced moderately fast, but the appearance of the second equivalent occurred with a rate constant at least two order of magnitude lower. However, for the perhydrolysis of oxalic esters with an excess of hydrogen peroxide, the simultaneous release of two equivalents of phenol was observed, with apparently the same rate constant. This fact indicates that the rate-determining step for this reaction was the first nucleophilic attack of the hydrogen peroxide to the carbonyl group of the oxalic ester and that the second attack appears to be faster. These observations support a mechanism involving the formation of an aryl hydroperoxy oxalate as intermediate which undergoes a second, much faster, intramolecular nucleophilic attack, leading to the formation of a cyclic HEI. ${ }^{33}$

Although the interest in the kinetics of the pathways leading to the formation of the HEI in the PO system was increasing, the complexity of this transformation remained a barrier towards a better understanding of the process. Alvarez and coworkers ${ }^{34}$ studied the PO system with TCPO $\left(7, \mathrm{R}_{1}=\mathrm{R}_{2}=\mathrm{R}_{3}=\mathrm{Cl}\right)$, hydrogen peroxide and triethylamine as catalyst and DPA as activator in anhydrous ethyl acetate. The authors observed a very complex kinetic behavior and, using a large set of kinetic expressions, were able to simulate the variation of the emission intensity with 
the reaction time for three triethylamine concentrations. The complexity of the kinetic expression led the authors to postulate a mechanism involving at least three HEIs, two (Scheme 4, X and Y) leading to chemiexcitation and one (Scheme 4, Z) unable to produce light emission. In this work, several structures were proposed for assignment of the possible intermediates $\mathrm{X}, \mathrm{Y}$ and $\mathrm{Z}$ as high-energy intermediates (HEI). However, this complex kinetic behavior might also be explained by an intricate competition between the initial reaction pathways, avoiding the postulation of a variety of different HEIs. ${ }^{34}$

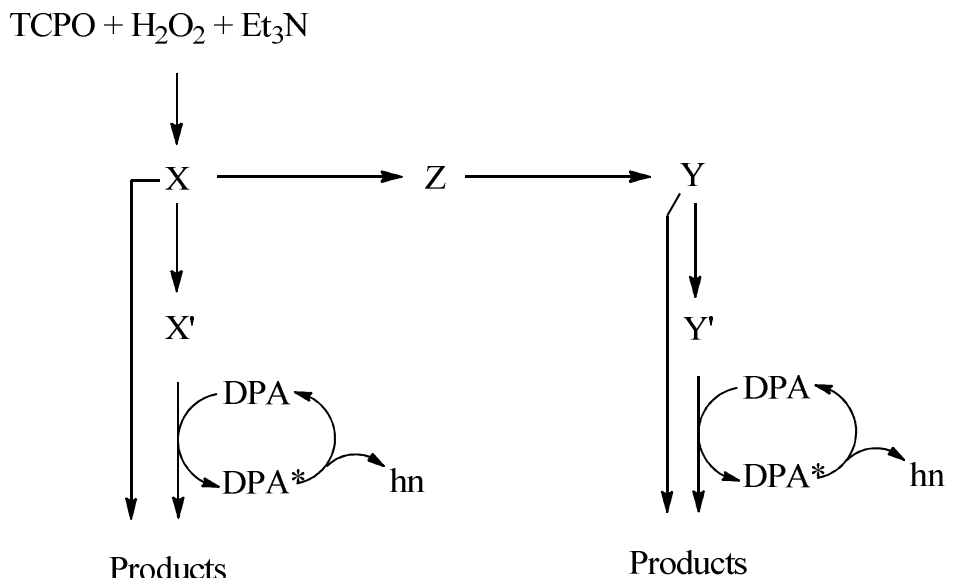

Scheme 4. Mechanistic pathway for HEI formation in the peroxyoxalate system, as proposed by Alvarez et al. ${ }^{34}$

In a subsequent study, Orlovic and coworkers ${ }^{35}$ used imidazole as catalyst for the PO reaction which was shown in a previous work to be a better catalyst than triethylamine. ${ }^{36}$ The kinetic behavior of the system in aqueous acetonitrile was significantly more complicated than in anhydrous media, demanding a much more complex set of kinetic expressions to be simulated. The system however had a simpler kinetic behavior with expressive water concentrations (25\% in acetonitrile), and the authors attributed the formation rate constant $\left(\mathrm{k}_{\mathrm{f}}\right)$ to the sum of two processes $\mathrm{k}_{\mathrm{r} 1}$ and $\mathrm{k}_{\mathrm{r} 2}$, which they attributed to the reaction of $\mathrm{H}_{2} \mathrm{O}_{2}$ and imidazole with the oxalic ester, respectively (Scheme 5). However, in a subsequent kinetic study on this system performed by our research group, where it was possible to obtain a simple kinetic behavior in anhydrous ethyl acetate, it has been shown that this attribution was not correct. ${ }^{37}$

A photoinitiated PO reaction was described by Milofsky and Birks, ${ }^{38}$ as an alternative to the classical PO reaction. Irradiation of an oxygenated solution of TCPO in the presence of a suitable activator in protic solvents with a laser at $295 \mathrm{~nm}$ led to the observation of a delayed light emission. The reaction mechanism is thought to involve light absorption by the oxalic ester, followed by hydrogen abstraction to form a radical species which reacts with dissolved oxygen, producing a peroxyl radical which can finally lead to the formation of a peracid intermediate, also formed as a crucial intermediate in the normal PO reaction. This reaction, named 
photoinitiated chemiluminescence (PICL), has been utilized to detect fluorescent polycondensed aromatic hydrocarbons.

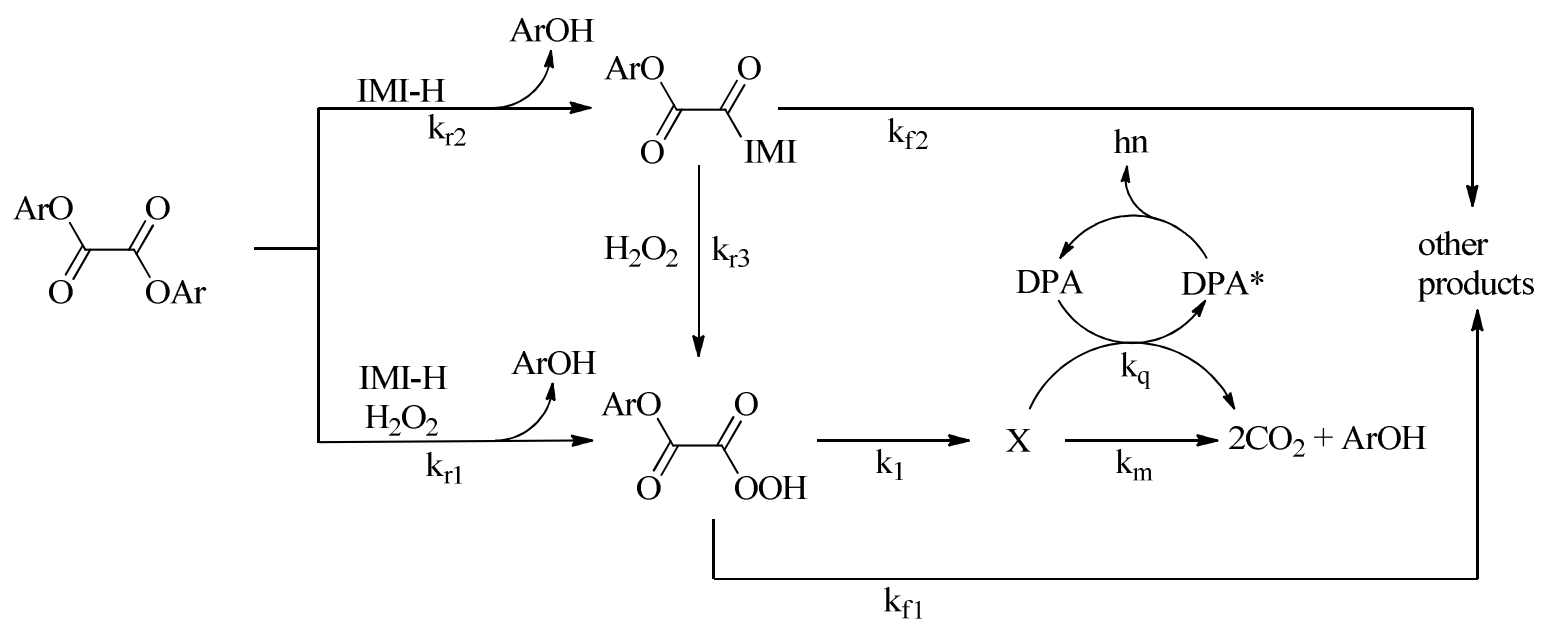

Scheme 5. Mechanism for the peroxyoxalate chemiluminescence of TCPO with imidazole (IMI$\mathrm{H})$ as catalyst, as proposed by Orlovic et al. ${ }^{35}$

Thereafter, Milofsky and Birks published another article about a mechanistic study on the PICL with the PO reaction, taking advantage of the fact that they excluded the necessity to add hydrogen peroxide to initiate the PO reaction, which could simplify the kinetic expression for the light emission. ${ }^{39}$ The authors studied this system in several experimental conditions, and were able to propose a reaction mechanism, including several speculative high-energy intermediate structures (see below).

In order to obtain more detailed information on the mechanism of base catalysis in the PO system, Neuvonen ${ }^{40}$ studied the kinetics of neutral and imidazole-catalyzed hydrolysis of bis(4nitrophenyl)oxalate (4-NPO), following the reaction progress by observing the 4-nitrophenol UV absorption at $310 \mathrm{~nm}$. The rate of neutral hydrolysis of 4-NPO in aqueous acetonitrile solution was shown to correspondent to the release of the second equivalent of 4-nitrophenol, since the release of the first equivalent appeared to be considerably faster. Using $\mathrm{D}_{2} \mathrm{O}$ instead of $\mathrm{H}_{2} \mathrm{O}$ as solvent led the author to suggest the $\mathrm{B}_{\mathrm{AC}} 3$ mechanism to be operating in the hydrolysis of the first ester group in 4-NPO. ${ }^{41}$ However, with imidazole as catalyst, the reaction is much faster and the rate constant refers to the release of the second 4-nitrophenol unit, being the first phenol released at least 10 times faster than the second one. Furthermore, a second-order dependence of the rate constants with the imidazole concentration was reported, and the formation of a transient intermediate absorbing in the region between 230 and $250 \mathrm{~nm}$ could be observed. These results suggested the initial formation of 1-aroxalylimidazole followed by formation of 1,1'oxalyldiimidazolide (ODI, 10) as a second reactive intermediate, consistent with a general basecatalyzed nucleophilic attack of imidazole on the oxalic ester in the initial steps of the PO reaction (Scheme 6). ${ }^{42}$ 


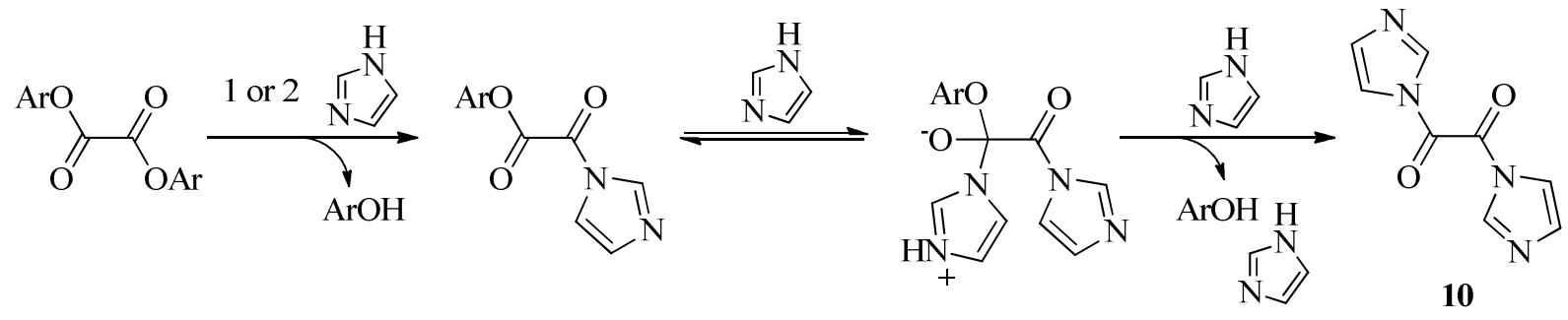

Scheme 6. Formation of the reactive intermediate ODI (10) in the PO reaction, as proposed by Neuvonen. ${ }^{40}$

Thereafter, Neuvonen studied in more detail the occurrence of ODI as a reactive intermediate in the reaction of TCPO and DNPO with imidazole, employing stopped-flow techniques, ${ }^{43}$ and outlined a mechanism similar to that proposed by Hadd and coworkers. ${ }^{44,45}$ This mechanism involved a pre-equilibrium step with imidazole addition to the carbonyl group and formation of a zwitterionic tetrahedral intermediate and indicated similar reactivity for both oxalic esters groups. A second imidazole molecule acts as a general-base catalyst in the deprotonation of the acylimidazolium cation inducing the release of a phenolate unit; thereby explaining the secondorder dependence of the rate constants on the imidazole concentration, observed previously. ${ }^{40}$

The role of ODI as reactive intermediate in the PO system was then investigated by Hadd and coworkers from Birks' research group ${ }^{46}$ in a systematic study of the chemiluminescence reaction kinetics for mixtures of ODI and hydrogen peroxide in the presence of an activator, using imidazole, lutidine and collidine as catalysts. Observing the reaction under pseudo first-order conditions, Hadd et al. suggested two possible pathways for light emission, one involving an imidazole-substituted peracid derivative as intermediate, that undergoes an intramolecular cyclization reaction to an imidazoyl-hydroxy-dioxetanone derivative as HEI; the other pathway implied in the formation of a much more complex intermediate structure with a six-membered ring peroxide intermediate. Apart from the unconfirmed proposal for the HEI structure, the authors were able to establish ODI as an intermediate in the PO reaction with oxalic esters and imidazole as catalyst. ${ }^{46}$

The kinetic of the reaction of TCPO with hydrogen peroxide catalyzed by imidazole was studied in details by Stevani and coworkers, ${ }^{37}$ which employed a wide range of imidazole concentrations and measured the reaction rates both by UV-Vis spectroscopy and by observing the light emission intensity. The authors suggested a mechanistic pathway that required the involvement of imidazole-substituted reactive intermediates, and also attributed experimentally obtained rate constants to three main reaction steps preceding the formation of the HEI. Bi- and trimolecular rate constants (containing first and second-order contributions of the imidazole concentration) were obtained for the first nucleophilic substitution on the oxalic ester by imidazole $\left(\mathrm{k}_{1}\right.$, Scheme 7$)$ and the substitution of the second phenolic unit by imidazole was shown to be much faster than the first one, not being kinetically observed ( $k_{2}$, Scheme 7$)$. From the peroxide dependence of one of the observed emission rate constants, it was possible to obtain 
the bimolecular rate constant for imidazole-catalyzed nucleophilic hydrogen peroxide attack to ODI $\left(\mathrm{k}_{3}\right.$, Scheme 7$)$ and roughly estimate the cyclization rate constant of the oxalic peracid derivative to the HEI ( $\mathrm{k}_{4}$, Scheme 7$)$. However, also in this study, as in all previous kinetic studies on the peroxyoxalate reaction, no kinetic data could be obtained with respect to the interaction of the HEI with an activator (ACT) leading to electronically excited state formation (Scheme 7). ${ }^{37}$

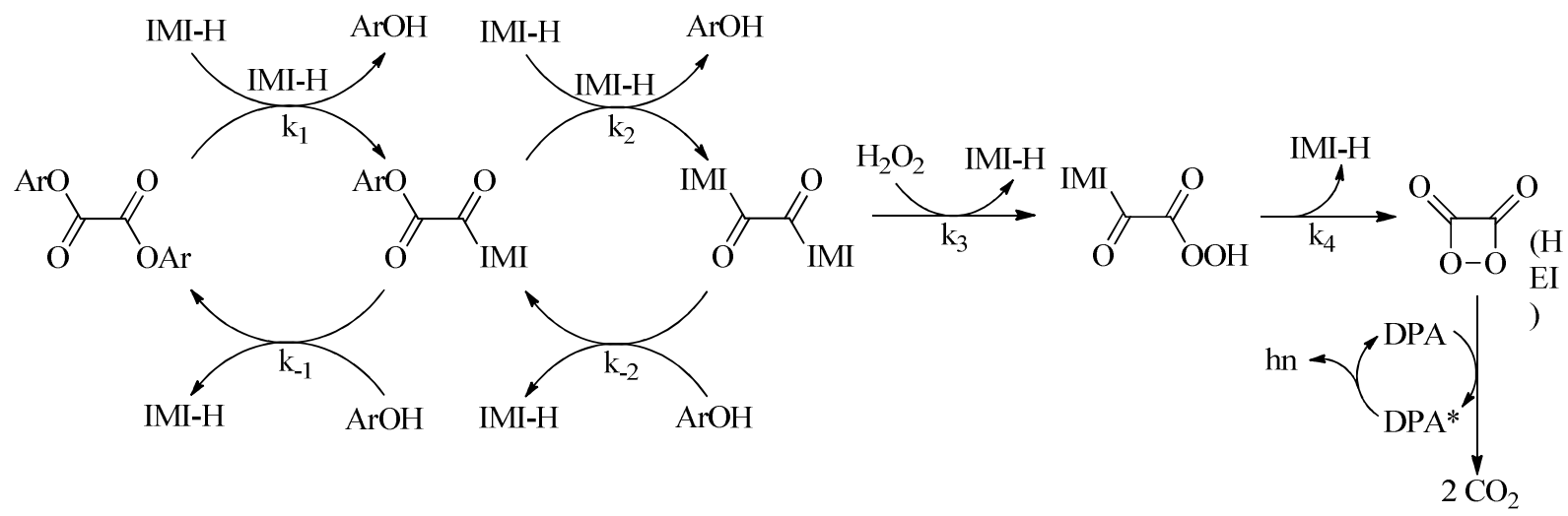

Scheme 7. Mechanistic proposal for the chemiluminescence of the peroxyoxalate system, as suggested by Stevani et al. ${ }^{37}$

In a subsequent work on this system, da Silva and coworkers ${ }^{47}$ were able to suggest a complete reaction sequence for the imidazole-catalyzed PO reaction which could explain the observed light-emission profile, consisting of an initial rapid increase of the emission intensity, followed by a slower decay (Figure 3). The reaction kinetics was studied using both, light emission intensity and absorption by produced phenol as parameters, and it was possible to attribute the faster rate constant of the rise in emission intensity (Figure $3, \mathrm{k}_{\mathrm{obs}} 2=0.13 \mathrm{~s}^{-1}$ ) to the bimolecular nucleophilic hydrogen peroxide attack on the intermediate ODI, as well as the slower emission intensity decay rate constant (Figure $3, \mathrm{k}_{\mathrm{obs}} 1=0.0035 \mathrm{~s}^{-1}$ ) to the replacement of the phenolic substituent at the TCPO carboxylic group by nucleophilic imidazole attack. The latter reaction step could also be observed in absorption experiments accompanying the liberation of the phenolic units. The reaction mechanism proposed in this work is in general agreement with mechanisms proposed earlier by Stevani et al. (Scheme 7), however, a more detailed study on the dependence of the emission intensity rate constants on the hydrogen peroxide concentration, allowed the determination of exact values for the cyclization rate constants at different imidazole concentrations, showing the role of imidazole as a base catalyst in this step and obtaining a bimolecular rate constant of $320 \mathrm{~mol} \mathrm{~L}^{-1} \mathrm{~s}^{-1}$ for this transformation; this considerably high rate constant could not be observed by traditional kinetic measurement. 


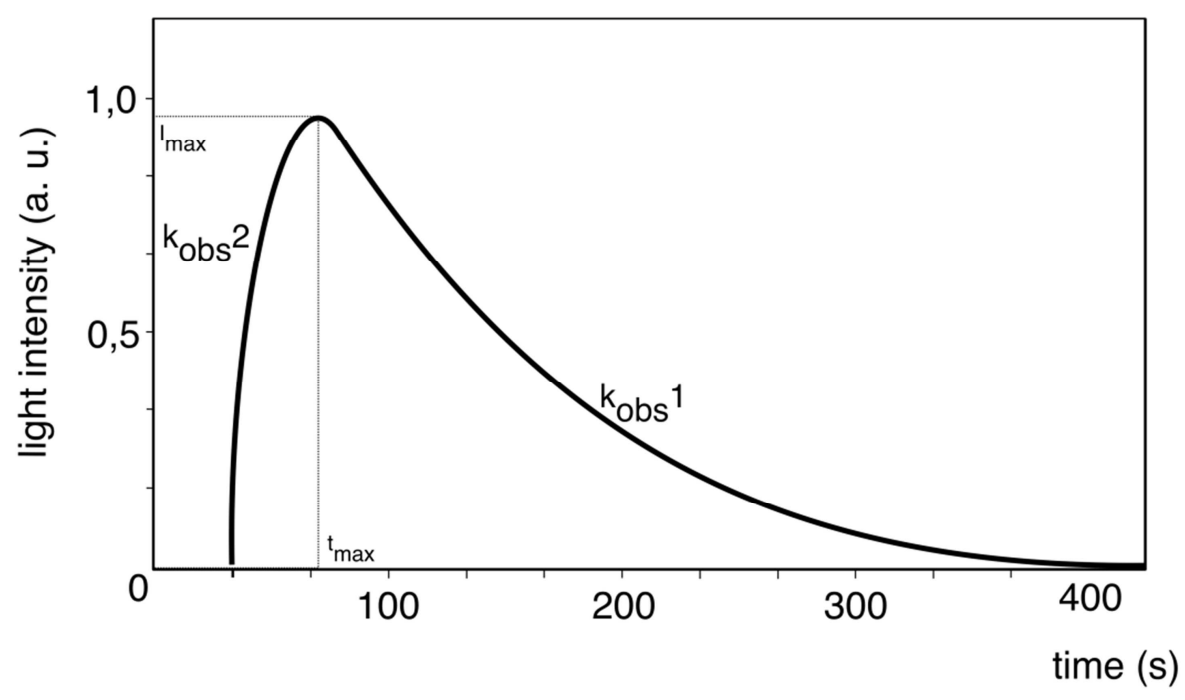

Figure 3. Emission intensity time course of peroxyoxalate chemiluminescence. $[\mathrm{TCPO}]=0.1 \mathrm{mM},\left[\mathrm{H}_{2} \mathrm{O}_{2}\right]=10.0 \mathrm{mM},[\mathrm{IMI}-\mathrm{H}]=1.0 \mathrm{mM}$ and $[\mathrm{DPA}]=1.0 \mathrm{mM}$, in anhydrous ethyl acetate at $25^{\circ} \mathrm{C}$.

In order to obtain a more precise insight into the reaction mechanism of the peroxyoxalate system, two research groups independently attempted the synthesis and study of peracid intermediates in the reaction sequence. Chokshi and coworkers ${ }^{48}$ performed a ${ }^{19} \mathrm{~F}$ NMR study on the reaction of bis(2,6-difluorophenyl)oxalate with hydrogen peroxide and concluded that the peracid derivative 11, should be an essential reactive intermediate in this transformation. Hohman and coworkers ${ }^{49}$, from the same research group, achieved the synthesis of silylprotected oxalic peracids $\mathbf{1 3}$ and $\mathbf{1 4}$ and studied their chemiluminescence properties, concluding that these compounds are involved in the reaction sequence leading to light emission. Light emission with a very fast decay rate was easily observed, upon addition of fluoride as deprotection agent to the silyl-protected oxalic peracids 13 and 14, in the presence of DPA.

In the same period of time, Stevani and coworkers ${ }^{50}$ reported the characterization and the chemiluminescence properties of 4-chlorophenyl- $O, O$-hydrogen monoperoxyoxalate (12). Kinetic studies on the reaction of $\mathbf{1 2}$ with various bases in the presence of DPA, resulting in bright light emission, confirmed the involvement of oxalic peracids in the reaction sequence. Additionally, these studies showed unequivocally that peracid derivatives were not able to act as HEIs, which led to the formation of electronically excited states upon its interaction with an activator (see also below). ${ }^{51}$ 


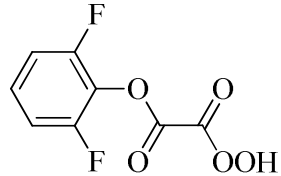

11

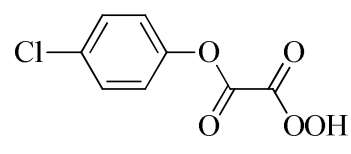

12
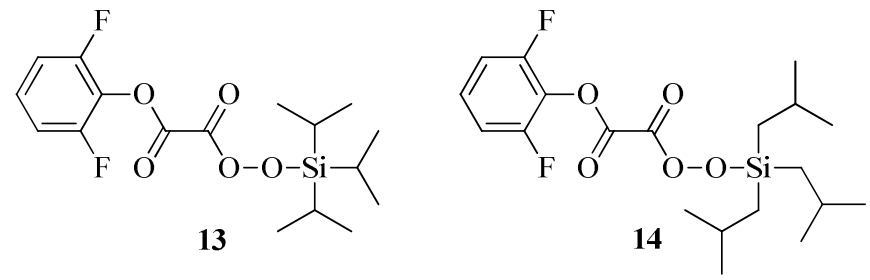

Da Silva ${ }^{52}$ and Lang $^{53}$ performed more detailed kinetic studies on the cyclization step of different monoperoxyoxalates, containing electronically distinct aromatic substituents. A Hammett correlation of the cyclization rate constants for several derivatives gave a Hammett reaction constant of $\rho=+2.4$, indicating the formation of a substantial negative charge on the phenolic leaving group in the transition state. Therefore, the cyclization step should occur in a concerted manner, with the intramolecular nucleophilic attack taking place simultaneously with the leaving of the phenolic group.

\section{Structure of the high-energy intermediate}

Already in the first study about the peroxyoxalate reaction, evidence has been obtained that the reaction of an oxalic derivative with hydrogen peroxide occurred with the formation of an intermediate, that was not itself the emitting species. Chandross ${ }^{24}$ initially proposed a possible pathway for the reaction of oxalyl chloride and hydrogen peroxide, including formation of a peracid intermediate. The exothermicity of the reaction, estimated to be about $70 \mathrm{kcal} \mathrm{mol}^{-1}$, was considered to be high enough for excited state generation, however not sufficient to excite $\mathrm{HCl}$, $\mathrm{CO}$ and $\mathrm{CO}_{2}$, the detected reaction products, of the reagent oxalyl chloride. Chandross also observed that vapors produced by the reaction had the property of inducing light emission from filter paper strips impregnated with fluorescent molecules, like anthracene, suggesting the existence of a metastable volatile species as the HEI, however, no structural proposal for this intermediate was made. $^{24}$

Rauhut and coworkers ${ }^{28}$ outlined a mechanistic scheme for the reaction of oxalyl chloride with hydrogen peroxide and initially proposed the monoperoxalic acid derivative $\mathbf{1 5}$ as a possible HEI, producing excited states from the energy of its fragmentation. In a following study on the reaction of oxalic anhydrides, Rauhut and coworkers ${ }^{29,54}$ suggested two other possible HEI structures: the cyclic peroxide 16 and its $\mathrm{HCl}$ elimination product 1,2-dioxetanedione (17), a dimer of carbon dioxide (Scheme 8). It was proposed that these cyclic peroxides could form excited states from the energy of an exothermic concerted cleavage process.

In subsequent studies on the reaction of oxalic ester with hydrogen peroxide, ${ }^{30}$ Rauhut and coworkers designed specific experiments to get insight into the nature of the HEI. One of these experiments includes the delayed addition of an ACT to the reaction medium, observing substantial emission even if the ACT addition was delayed as much as 70 minutes, while in the same conditions, with the ACT added from the beginning, no more emission could be observed 
after this reaction time. Those results led to the conclusion that an intermediate was indeed formed in the course of the reaction, stable enough to be accumulated, which was however rapidly destroyed when mixed with the ACT. The second experimental setup was to conduct the reaction of DNPO with hydrogen peroxide in dimethylphtalate and pass a stream of gas through the reaction mixture, trying to observe if an intermediate could be volatile and stable enough to excite the ACT in another flask. It was observed that the gas stream, irrespective of the carrier gas used, was capable of generating bright, short-lived chemiluminescence, when in contact with an ACT solution. This result indicated the volatile and meta-stable nature of the HEI, however, attempts to characterize it in a gas-phase infrared spectrometer or a mass spectrometer failed, only being able to detect the final reaction product carbon dioxide. ${ }^{30}$

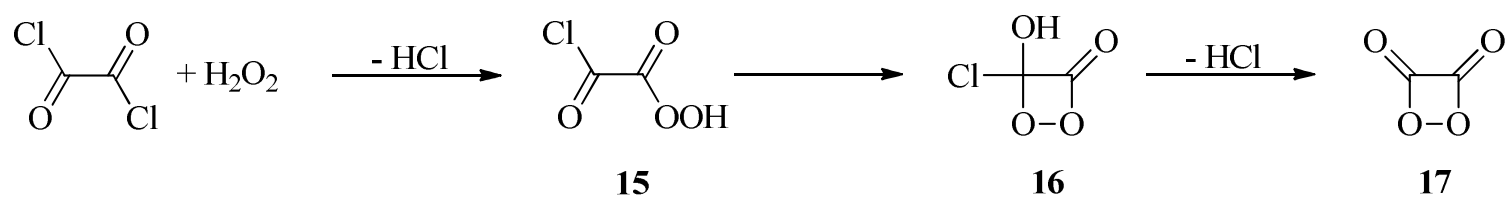

Scheme 8. Mechanism for the reaction of oxalyl chloride with hydrogen peroxide, showing the formation of a possible HEI, as proposed by Rauhut. ${ }^{28}$

Cordes and coworkers ${ }^{55}$ attempted the detection of 1,2-dioxetanedione in a mass spectrometer, carrying out the same reaction but using a different experimental arrangement, being able to detect peaks of $m / z 17,18,28,32,34,44,60$ and 88. The peak at 44 was attributed to $\mathrm{CO}_{2}$, the one at 60 to $\mathrm{CO}_{3}$ and the 88 peak to $\mathrm{C}_{2} \mathrm{O}_{4}$, the molecular formula of 1,2dioxetanedione. Following the peak intensity at $\mathrm{m} / \mathrm{z}$ at 88 with time, the authors described a decrease in the signal intensity that eventually reached the background level. To test the system, the mass spectrometer was replaced by a glass tube containing a paper strip impregnated with 9,10-bis(phenylethynyl)anthracene, and light emission could be observed, confirming that the HEI was indeed being conducted to the mass spectrometer analysis chamber.

The assumption that the 1,2-dioxetanedione was the HEI seemed to be experimentally confirmed. However, De Corpo and coworkers ${ }^{56}$ repeated the same reaction but with a mass spectrometer set-up with variable excitation and residence time, and an experimental arrangement favoring the direct injection of reaction products without the need of a metal needle. The authors reported indeed a signal at $m / z$ 88, but studying the rise of this peak in function of the residence time in the instrument chamber, they observed that the substance was being actually formed in the instrument, not consumed as expected for a reactive intermediate. The source for the peak at $m / z, 88$ seemed to be a product of a reaction between the ion $\mathrm{CO}_{2}{ }^{+}$and the ester or between $\mathrm{RCOO}^{+}$and $\mathrm{CO}_{2}$, forming a metastable ion $\mathrm{C}_{2} \mathrm{O}_{4}{ }^{+}$not derived from the 1,2dioxetanedione. On the basis of these results, it should be noted that there is no direct mass spectrometric evidence for the occurrence of 1,2-dioxetanedione as intermediate in the peroxyoxalate reaction. 
Even without direct evidence for its existence, 1,2-dioxetanedione has been postulated by many authors as intermediate in the peroxyoxalate reaction, due to the overall of experimental evidence obtained throughout the years. Orosz and coworkers ${ }^{57}$ observed the kinetics of carbon dioxide release, following its IR absorption band at $2336 \mathrm{~cm}^{-1}$ in the reaction of DNPO with hydrogen peroxide in the presence of rubrene, concluding that the same HEI was responsible for light emission and also non-emissive $\mathrm{CO}_{2}$ formation. Additionally it was observed that the same intermediate is formed in the reaction of oxalyl chloride as well as oxalate esters with hydrogen peroxide, indicating 1,2-dioxetanedione as HEI structure.

In a search for new analytical applications of the PO system for the detection of aromatic hydrocarbons, Sigvardson and Birks ${ }^{58}$ observed a consistent background light emission from the reaction mixture in the absence of any ACT, as already reported before by the groups of Rauhut $^{28}$ and Weinberger. ${ }^{59}$ Additionally, Mann and Grayeski ${ }^{60}$ performed a more detailed study on this background emission, correlating it to the formation and thermal decomposition of the HEI. The broad unstructured emission spectrum with a maximum at around $450 \mathrm{~nm}$, obtained with all oxalic esters utilized in the reaction, led the authors to postulate that the HEI should not contain any aromatic substituent, indicating 1,2-dioxetanedione as the most probable HEI. The observed emission was attributed to the singlet or triplet excited state of a bent $\mathrm{CO}_{2}$ molecule, since these possess energy levels at 32000 and $46700 \mathrm{~cm}^{-1}$ above the ground state respectively. The authors thereby excluded the possibility, that this emission could be originated from the singlet or triplet excited state of 1,2-dioxetanedione, as suggested earlier by Stauff and coworkers. $^{61}$

Studying the complex kinetics of the reaction of TCPO with triethylamine and hydrogen peroxide, in the presence of DPA and in ethyl acetate as solvent, Alvarez and coworkers ${ }^{34}$ suggested that at least two HEI ( $\mathbf{X}$ and $\mathbf{Y}$ ) which are capable of generating chemiluminescence emission, should be present in the PO system, with at least one more intermediate $(\mathbf{Z})$ unable to generate light emission. Compound $\mathbf{Z}$ was postulated to occur as an intermediate in the transformation between the two proposed HEI species $\mathbf{X}$ and $\mathbf{Y}$ (see Scheme 4). The authors suggested structures for those intermediates, speculating that $\mathbf{X}$ could be like $\mathbf{1 8}$ or $\mathbf{1 9}, \mathbf{Z}$ like $\mathbf{2 0}$ and $\mathbf{Y}$ like 21 or 1,2-dioxetanedione (17). However, these suggestions for the HEI structure, similarly to those made by Orosz, were just based on kinetic data and no direct structural evidence has been obtained for the validity of the proposal. In subsequent studies, Stevani and coworkers $^{50}$ isolated the 4-chlorophenyl- $O, O$-hydrogen monoperoxyoxalate (12) and showed that this peroxide did not interact directly with activators like DPA, as $\mathbf{1 2}$ proved to be stable in the presence of DPA and no light emission could be observed. This experimental result unequivocally excludes peracid derivatives like 12, as well as 15 and 19 as HEIs in the peroxyoxalate reaction. However, when a base was added to the mixture of $\mathbf{1 2}$ and DPA, a burst of bright light emission was observed, confirming the involvement of oxalic peracids in the peroxyoxalate reaction sequence, however, not as HEIs. ${ }^{50}$ 


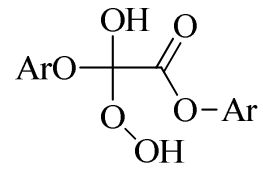

18<smiles>O=C(OO)C(=O)O[Al]</smiles>

19

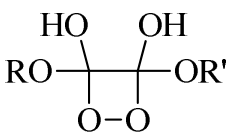

$\mathrm{R}, \mathrm{R}^{\prime}=\mathrm{H}, \mathrm{Ar}$

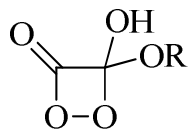

$\mathrm{R}=\mathrm{H}, \mathrm{Ar}$

20

21

The study of the photoinitiated peroxyoxalate reaction ${ }^{39}$ led to the proposal of the complex cyclic peroxides 22, 23 and 24 as possible candidate for the HEI structure, again based on indirect kinetic evidence. The study of Hadd and coworkers ${ }^{46}$ on the reaction of TCPO with hydrogen peroxide catalyzed by imidazole in anhydrous media led to the suggestion of structure 25, which could be transformed into 1,2-dioxetanedione, as HEI, as well as structure 26, believed to be important at high imidazole concentrations. Those structures were also suggested as HEIs by Lee and coworkers, ${ }^{62,63}$ which supported the kinetic interpretation of Hadd. However, Lee and coworkers $^{64}$ considered $\mathbf{2 5}$ and related structures with different bases instead of imidazole as the most probable HEIs, which were expected to show significantly different kinetic properties depending on the particular base utilized.

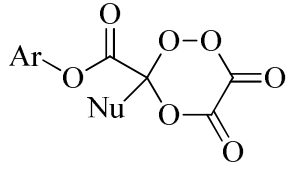

22

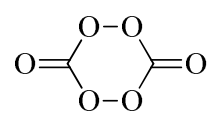

23

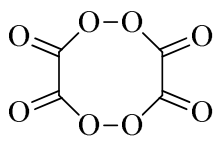

24

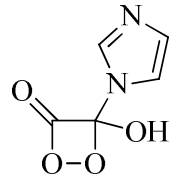

25

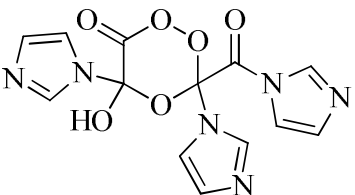

26

In a different approach, Stevani and Baader ${ }^{65}$ attempted trapping of the HEI intermediate by its reaction with triphenylantimony, which is known as a reagent capable of forming stable insertion compounds with cyclic peroxides, even with the more stable 1,2-dioxetanes. ${ }^{66}$ If the reaction of TCPO and $\mathrm{H}_{2} \mathrm{O}_{2}$ would occur with the formation of 1,2-dioxetanedione as HEI, this cyclic peroxide should be transformed to the 2,2,2-triphenyl-2 $\lambda^{5}$-1,3,2-dioxastibolane-4,5-dione (27) by its insertion reaction with triphenylantimony (Scheme 9). The authors transferred the vapors generated from the reaction of TCPO and $\mathrm{H}_{2} \mathrm{O}_{2}$ into another flask containing triphenylantimony; but no 27 formation could be observed. However, this experimental result does not exclude 1,2-dioxetanedione as a possible HEI, because this compound might be too unstable in the conditions tested to persist the time to reach the flask containing triphenylantimony, or, alternatively, catalytic decomposition of the HEI, without formation of the insertion product, might be the main reaction pathway. ${ }^{65}$ 

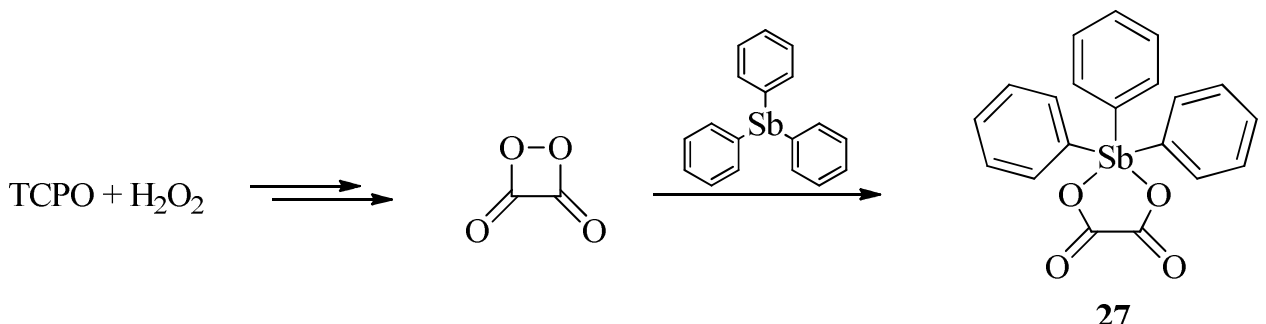

Scheme 9. Reaction of 1,2-dioxetanedione with triphenylantimony forming the stable insertion compound 27. ${ }^{65}$

Using spectroscopic methods, Bos et $a .^{67}$ apparently detected and confirmed 1,2dioxetanedione as the HEI of the peroxyoxalate system. The authors conducted studies monitoring the reaction of doubly ${ }^{13} \mathrm{C}$ labeled oxalyl chloride with anhydrous hydrogen peroxide in dry THF with DPA by ${ }^{13} \mathrm{C}$ NMR spectroscopy at low temperatures. They observed a singlet peak at $\delta 154.5 \mathrm{ppm}$ and assigned it to a symmetrical intermediate, attributed in the work as the 1,2-dioxetanedione, a suggestion supported by ab initio quantum mechanics calculations. Several other minor peaks appeared in the spectrum, and among them, some were attributed to an unsymmetrical intermediate that could not be identified and others as peaks of $\mathrm{CO}$ and $\mathrm{CO}_{2}$. Afterwards, the same group published a work ${ }^{68}$ concerning the identification of those other intermediates using 2D EXSY ${ }^{13} \mathrm{C}$ NMR spectroscopic techniques, in some respect supporting the possible intermediate $\mathbf{2 4}$ of the $\mathrm{PO}$ reaction proposed earlier by Birks. ${ }^{46}$ All those experiments were carried out at low temperatures $(<200 \mathrm{~K})$ due to the expected low thermal stability of the intermediates. However the reaction between oxalyl chloride and hydrogen peroxide was conducted, surprisingly, in the presence of DPA, which actually reacts with high rate constants with the HEI present in the reaction mixture, leading to a decrease in the HEI concentration. Since the spectroscopic data for the structure of the HEI were not correlated to the chemiexcitation properties, there is no unequivocal evidence that the intermediates detected are those responsible for chemiexcitation by reacting with an activator.

\section{Interaction of the high-energy intermediate with the activator}

In the initial study conducted by Rauhut and coworkers, ${ }^{28}$ the PO reaction between oxalyl chloride and hydrogen peroxide in diethyl ether was performed using two different activators, 9,10-diphenylanthracene (DPA) and perylene (PER), and the emission spectra from the chemiluminescent reactions were compared with the fluorescence spectra of the two compounds, determined in similar conditions. A correspondence between the spectra was observed, demonstrating that the light emission in the PO system was due to the formation of the singlet excited state of an added activator and its subsequent fluorescence emission. This result also excluded the participation of a direct emission from an excited carbon dioxide molecule. 
Additionally, the authors were able to exclude the participation of ground-state complexes of the activator with either oxalyl chloride or hydrogen peroxide in the emission mechanism of the system. The involvement of the endoperoxide of DPA, a known chemiluminescent compound, ${ }^{69}$ was also tested, by the addition of this compound to the reaction mixture, in the absence of DPA, not being able to detect any significant emission, thereby excluding also the DPA endoperoxide as an intermediate in the light emission reaction. ${ }^{28}$ Additionally, using traditional photophysical tools, the Rauhut group was able to verify that excitation of the ACT could not be due to an electronic energy transfer from an excited species, formed during the reaction, to the ACT. ${ }^{28}$ Furthermore, the authors observed a strong dependence of the PO reaction quantum yields on the structure of the activator, however with no effect on the observed reaction rates, showing that the chemiexcitation step - conversion of chemical energy into electronic excitation energy - was not the rate-determining step of this complex process. In this pioneer work, the Rauhut group had already observed that quantum yields could be correlated to the activator's oxidation potential and postulated the formation of a charge-transfer complex between HEI and the ACT during the transformation, furthermore, speculated on the involvement of charge transfer reactions in the chemiexcitation mechanism. $^{28}$

The energetics of the chemiexcitation step in the PO reaction was studied in detail by Lechtken and Turro, ${ }^{70}$ comparing the efficiency of the peroxyoxalate reaction with that of 3,3,4,4-tetramethyl-1,2-dioxetane (TMD) thermolysis. ${ }^{71}$ The authors used several fluorophores (activators) of different singlet energies in both chemiluminescence systems, in order to estimate the highest excitation energy available for singlet excited state generation. The TMD thermolysis was able to excite, with considerable quantum yields, molecules with singlet energies up to 84 $\mathrm{kcal} \mathrm{mol}^{-1}$, however, very low quantum yields were obtained for fluorophores with higher singlet energies. This behavior is typically for the occurrence of an electronic energy transfer from singlet excited acetone, formed during TMD thermolysis, to the fluorophore. Contrarily, the PO system showed a distinct behavior; the excitation efficiency proved to gradually diminish as the singlet energy of the added fluorophore increased. The maximum energy available from the PO system was estimated to be as high as $105 \mathrm{kcal} \mathrm{mol}^{-1}$. This indicates that the mechanism operating in the chemiexcitation step of PO chemiluminescence is completely different from that occurring for excited state formation in 1,2-dioxetane thermolysis, therefore excluding the occurrence of an electronic energy transfer excitation to the ACT in the PO system. The authors concluded from this study that chemiexcitation in the PO system in the presence of energy acceptors, is unlikely to occur by energy transfer from singlet excited $\mathrm{CO}_{2}$ to the activator, while fluorophore excitation probably occurs directly during the chemiexcitation process. Additionally, it could be verified in this work that the PO system does not involve triplet excited state formation. $^{70}$

Catherall and coworkers ${ }^{72}$ performed a systematic study of the reaction of bis(pentachlorophenyl)oxalate (PCPO) and hydrogen peroxide with several fluorophores, and observed a linear correlation, in a Stern-Volmer type relationship of $1 / \Phi_{\mathrm{CL}} v s$. $1 /[\mathrm{ACT}]$. Additionally, a linear correlation was found between the chemiluminescence quantum yields and 
the ACT's oxidation potential, however, such a correlation was not obtained with the ACT's singlet energy, their fluorescence quantum yield or singlet state lifetimes. This work presented evidence that electron-exchange reactions between appropriate acceptors and donors were involved in the formation of excited states, although no further studies were conducted to characterize those interactions.

From these early studies on the chemiexcitation mechanism in the peroxyoxalate system it became evident that this transformation should involve a mechanism similar to the Chemically Initiated Electron Exchange Luminescence (CIEEL) mechanism, proposed earlier by Schuster ${ }^{13}$ for the catalyzed decomposition of isolated organic peroxides. As outlined above, the CIEEL hypothesis was formulated based on the reaction of an organic peroxide and fluorophores, that were renamed as "activators" (ACT), since the rate of the process was shown to depend on the concentration of this compound, showing that they did not just passively accept the excitation energy, but they did play an active role in the excited state generation mechanism.

While there are many known, highly efficient intramolecular chemiluminescence systems postulated to involve the CIEEL mechanism, ${ }^{9,11,19,21-23}$ the PO reaction would be the only known example of a chemiluminescence transformation with high quantum yields which is supposed to involve the intermolecular CIEEL mechanism in its chemiexcitation step. Stevani et al. studied the mechanism of the chemiexcitation step in peroxyoxalate chemiluminescence using several activators, including polycondensed aromatic hydrocarbons ${ }^{73}$ and highly fluorescent steroid derivatives. ${ }^{74}$ The emission quantum yields for the PO system obtained with several activators were correlated to the ACT concentration by means of a double-reciprocal plot of $1 / \Phi_{\mathrm{CL}} v s$. $1 /[\mathrm{ACT}]$, allowing calculation of the ratio between the rate constant of electron transfer from the ACT to the HEI $\left(\mathrm{k}_{\mathrm{CAT}}\right)$ and the rate of dark decomposition of the HEI $\left(\mathrm{k}_{\mathrm{D}}\right)$ (Scheme 10).

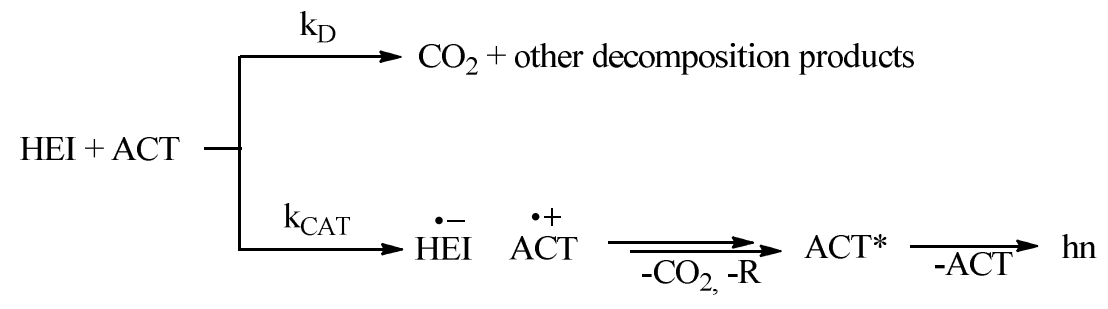

Scheme 10. Basic mechanistic scheme for the interaction of a high-energy intermediate (HEI) with an activator. (i) Dark decomposition of the HEI $\left(\mathrm{k}_{\mathrm{D}}\right)$; (ii) light production by interaction of the HEI with the ACT $\left(\mathrm{k}_{\mathrm{CAT}}\right)$, according to the CIEEL mechanism.

This methodology allows to obtain rate constants, although only relative ones, for the interaction of the HEI with the ACT, a very fast reaction which could not be observed by standard kinetic measurements. This ratio $\mathrm{k}_{\mathrm{CAT}} / \mathrm{k}_{\mathrm{D}}$ was used to compare the $\mathrm{k}_{\mathrm{CAT}}$ values for different ACTs, since the values for $\mathrm{k}_{\mathrm{D}}$ should remain unchanged as the reactions conditions were maintained. A linear plot with slope $\alpha=0.13 \pm 0.2$ was observed in the linear free-energy 
correlation between $\ln \left(\mathrm{k}_{\mathrm{CAT}} / \mathrm{k}_{\mathrm{D}}\right)$ and the half-wave oxidation potential of the ACTs, as expected for a mechanism involving an electron transfer or at least a charge transfer from the ACT to the peroxide in its rate-determining step. ${ }^{73,74}$ These results clearly indicate the occurrence of the CIEEL mechanism in the chemiexcitation step of the peroxyoxalate reaction, involving an electron transfer process with an early transition state, as suggested by the relatively low value for the electron transfer coefficient $(\alpha)$.

According to the CIEEL mechanism for the interaction of 1,2-dioxetanedione, the most probable HEI in the peroxyoxalate system, and DPA (Scheme 11), the elementary steps leading to the formation of electronically excited states and subsequent light emission are: a) formation of a charge-transfer complex between the peroxide and the activator $\left(\mathrm{K}_{\mathrm{CT}}\right)$; b) electron transfer from the DPA to the 1,2-dioxetanedione $\left(\mathrm{k}_{\mathrm{ET}}\right)$, facilitated by the elongation of the $\mathrm{O}-\mathrm{O}$ bond by thermal activation, which lowers the energy level of the antibonding orbital of this bond, occurring probably simultaneous with $\mathrm{O}-\mathrm{O}$ bond cleavage, enabling the energetically unfavorable electron transfer by making it essentially irreversible; c) cleavage of the $\mathrm{C}-\mathrm{C}$ bond and formation of a neutral carbon dioxide fragment and a carbon dioxide radical anion $\left(\mathrm{k}_{\mathrm{CLEAV}}\right)$, still within the solvent cavity with the DPA radical cation; d) electron back-transfer (EBT) from the carbon dioxide radical anion to the ACT radical cation $\left(\mathrm{k}_{\mathrm{EBT}}\right)$, an annihilation process liberating enough energy for the formation of DPA in its first singlet excited state; e) decay of singlet excited DPA to its ground state, producing DPA fluorescence emission (Scheme 11).

Since the discovery of the PO system it has been postulated that, under certain reaction conditions, an intermediate should be capable of accumulating and leading to light emission upon fast reaction with an ACT added after a certain reaction time. However, this observation could not be reproduced by other authors and was actually challenged since it was supposed that the HEI would be too labile to be accumulated even in a thoroughly purified reaction medium. ${ }^{72}$ However, very recently, Ciscato and coworkers ${ }^{75}$ were able to observe the accumulation of a HEI in the reaction of oxalyl chloride and hydrogen peroxide in a highly purified ethyl acetate as solvent, in certain reaction conditions. Furthermore, it was shown to be possible to measure the rate constant of the interaction between the HEI and several activators $\left(\mathrm{k}_{\mathrm{CAT}}\right)$, added with a delay in time to the system. The linear free-energy correlation between the rate constants and the activator's oxidation potential ( $\ln \mathrm{k}_{\mathrm{CAT}} v s$. $\mathrm{E}_{\mathrm{ox}}$ ) led to an $\alpha$ value of $0.23 \pm 0.02$, pointing also to an early transition state with respect to electron transfer and presumably also to $\mathrm{O}-\mathrm{O}$ bond cleavage, as these two steps are supposed to occur concomitantly. This $\alpha$ value is in agreement with the values reported for other CIEEL systems, including decomposition of cyclic peroxides, typically in the range $0.1-0.3$. The reaction rates for the interaction between the HEI and the activators showed to be very high, reaching rate constant values of up to $1.7 \times 10^{5} \mathrm{~L} \mathrm{~mol}^{-1} \mathrm{~s}^{-1}$ for the case of rubrene, showing the extremely high reactivity of the HEI with activators. For DPA, a slower however still considerably high rate constant of $3.7 \times 10^{3} \mathrm{~L} \mathrm{~mol}^{-1} \mathrm{~s}^{-1}$ was measured, and for this activator the activation parameters were also determined as: $\Delta \mathrm{H}^{\neq}=4.2 \pm 0.1 \mathrm{kcal} \mathrm{mol}^{-1}$, $\Delta \mathrm{S}^{\neq}=-26.9 \pm 0.2 \mathrm{cal} \mathrm{mol}^{-1} \mathrm{~K}^{-1}$ and $\Delta \mathrm{G}^{\neq}$at $298 \mathrm{~K}=12.2 \pm 0.1 \mathrm{kcal} \mathrm{mol}^{-1}$, for [DPA] $=1.0 \mathrm{mmol}$ $\mathrm{L}^{-1}$. Therefore, these experimental results provide for the first time direct unequivocal evidence 
for the occurrence of an electron transfer in the chemiexcitation step of the peroxyoxalate system, indicating the validity of the CIEEL mechanism for this transformation.

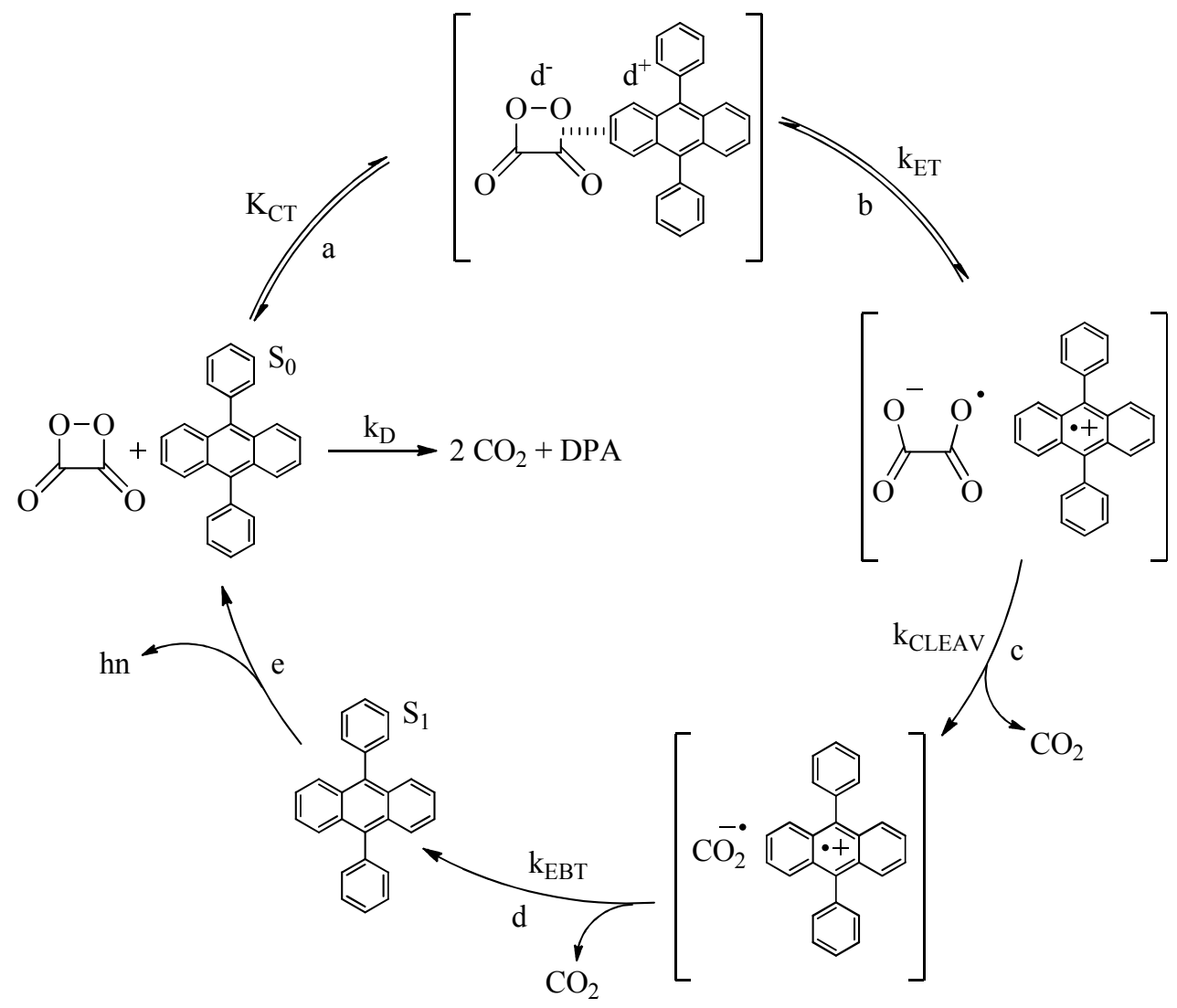

Scheme 11. Interaction of 1,2-dioxetanedione with 9,10-diphenylanthracene in the chemiexcitation step of the PO reaction, according to the Proposed CIEEL mechanism.

The above outlined results clearly indicate the occurrence of an electron transfer or at least a substantial charge-transfer from the activator to the peroxidic intermediate in the rate-limiting step of the peroxyoxalate chemiexcitation, based on indirect and direct kinetic evidence. However, in the former work it was also verified that activators bearing electron-withdrawing substituents, therefore possessing substantially high oxidation potentials, show high rateconstants for their interaction with HEI, therefore violating the linear free-energy correlation obtained with other activators. ${ }^{73}$ This unexpected observation encouraged Bartoloni and coworkers $^{76}$ to perform a systematic study where the relative catalytic rate constants and singlet quantum yields in the peroxyoxalate system were measured using 9-chloro, 9,10-dichloro, 9cyano and 9,10-dicyanoanthracene as activators. The linear free-energy relationship of the relative rate constants with the activators' reduction potentials $\left(\mathrm{E}_{\mathrm{red}}\right)$ confirmed, for the first time, the occurrence of an inverse electron transfer in the chemiexcitation step; in this case the electron transfer occurs from the HEI to the activator. In this inverse CIEEL process, once the pair of 
radical ions, with an inverse charge distribution as compared to the normal CIEEL mechanism, is formed, the electron back-transfer (from the radical anion of the ACT to a carbonyl radical cation) may also be capable singlet excited state formation, however, with low excitation quantum yields. ${ }^{76}$ An additional evidence for the validity of the occurrence of the inverse CIEEL mechanism for electron acceptor-substituted activators has been obtained by the observed correlation between the measured excitation quantum yields and the energy liberated in the chemiexcitation step, calculated from the redox-potentials of the radical-ions involved, ${ }^{76}$ in analogy to the correlation obtained before between the quantum yields in the "normal" CIEEL process and the energy liberated upon annihilation between the carbon dioxide radical anion and the radical cations of the commonly utilized activators. ${ }^{73}$

\section{Analytical applications of peroxyoxalate chemiluminescence}

A search in the Web of Science database (august 2011) over the past ten years, using the subjects "peroxyoxalate" and "analytical", reveals a total of 47 articles published with these keywords which have been cited 541 times, assuming an average number of 11.51 citations. The h-index of 14 for these articles confirms the general importance of the peroxyoxalate system for analytical and bioanalytical applications.
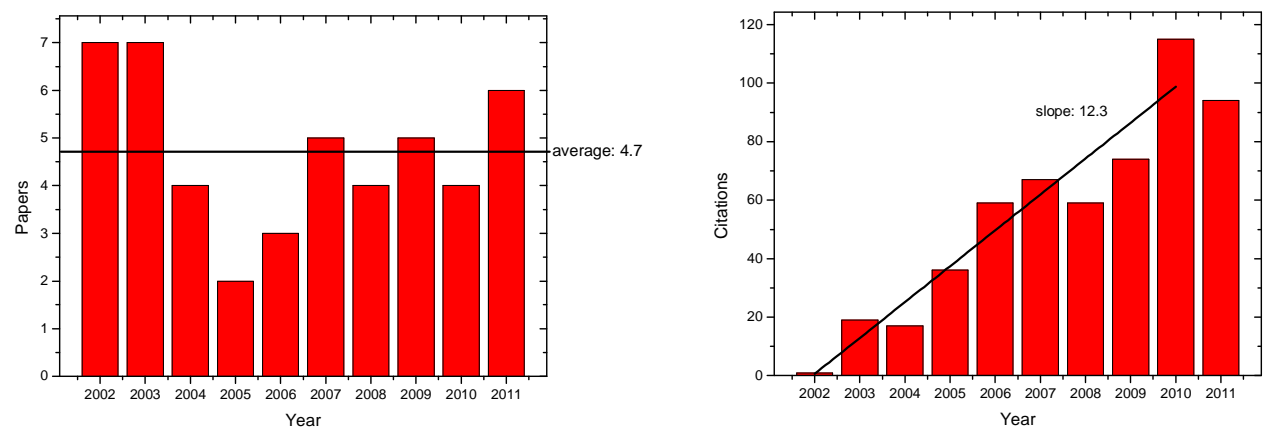

Figure 4. Evolution of articles published each year with the topics "peroxyoxalate" and "analytical" (left) and evolution of citation by year of these articles (right).

The number of articles published each year is approximately constant, with an average value of 4.7 papers per year (Figure 4, left), this shows that the applications of this system have been developed with a solid rate and indicates its consolidation as a technique of choice for many analytical assays. As expected, the number of citations is growing with the years, with an approximate rate of 12.3 citations per year (Figure 4, right). This number indicates an increase in the relevance of the subject, as its impact is rising with the years. If this tendency in the number of papers and citations is maintained, 2011 will be the most relevant year so far (Figure 4, right). Several recent reviews are concerned with analytical applications of chemiluminescence in general, including the peroxyoxalate system, ${ }^{77-79}$ however, one recent review is dedicated 
specifically to application of the peroxyoxalate reaction, including its utilizations together with techniques like high performance liquid chromatography, flow injection systems and capillary electrophoresis. ${ }^{77}$ The use of micelles, reversed micelles and microemulsions as reaction medium for the peroxyoxalate chemiluminescence has also been reviewed and the simplicity and low cost as well as the high sensitivity of this methodology were emphasized. ${ }^{81}$ The authors have shown the application of micellar peroxyoxalate chemiluminescence to detect fluorescent compounds in the nanomolar concentration range. Using reverse micelles it has been shown that ascorbic acid, rhodamine B and 8-anilino-1-naphthalenesulfonic acid, can be determined with detection limits in the order of $10 \mathrm{nM}^{81}$

In a general way, peroxyoxalate chemiluminescence can be utilized for the highly sensitive detection of any fluorescent compound, being able to substitute any assay based on fluorescence spectroscopy, with the advantage of avoiding problems of competitive absorption and scattering of excitation light, being therefore advantageous for biological and ambiental analysis. ${ }^{11}$ In some cases, specifically when using the peroxyoxalate reaction as detection system for high-pressure liquid chromatography and capillary electrophoresis, the fluorescent compound might be formed by post-column derivatization prior to detection, as is the case for substances like amino acids, carboxylic acids, carbonyl compounds, amines, catecholamines and many other compounds. ${ }^{82}$ As any fluorescence-based assay, peroxyoxalate CL can also be utilized for the quantitative determination of compounds which quench the fluorescence emission, although there are much less know examples for this application. ${ }^{83}$ In principle, the PO-CL could be utilized for the determination of basic catalysts which increase the reaction rate; however, no practical application has been addressed to this principle, although the reaction has been utilized for quantification of some metal ions, which apparently aggregate with basic catalysts. ${ }^{84}$ The reaction has been widely utilized for the detection of hydrogen peroxide under a variety of reaction conditions, ${ }^{85}$ and can also be utilized for the quantification of systems which produce or destroy hydrogen peroxide. ${ }^{86}$ Furthermore, the PO-CL has also been used for the determination of antioxidant activity. Finally, the system could be utilized in principle for the quantification of the chemiluminescent substrate, oxalic acid derivatives; however, to the best of our knowledge, this analysis principle has only been utilized for the quantification of oxalic acid itself in biological samples, ${ }^{87}$ as described in more details at the end of this article.

In the final part of this article, we would like to give some specific examples of recent analytical application of the PO-CL. For example, the use of 1,1'-oxalyldimidazole and 1,1'oxalyldi-4-methylimidazole instead of the aromatic oxalic esters in PO-CL has been studied and the effect of some imidazole derivatives (as 2-methyl- and 4-methylimidazole) as nucleophilic catalysts on the CL efficiency verified. ${ }^{64}$ Using 1,1'-oxalyl-4-methylimidazole as substrate instead of TCPO, higher maximum emission intensities and shorter reaction times have been obtained, a condition which might be useful for several analytical applications. One possible application of this methodology is the quantification of compounds like 1-aminopyrene, as this class of compound has a high environmental impact. ${ }^{64}$ 
An analytical method for the determination of the ability of antioxidants like $\beta$-carotene, $\alpha$ tocopherol, quercetin and L-ascorbic acid to scavenge hydrogen peroxide has been developed using the peroxyoxalate system with 9,10-diphenylanthracene as ACT in ethyl acetate/acetonitrile 9:1 as solvent. ${ }^{88}$ The PO-CL has also been applied for the determination of derivatized steroids in biological matrices, enabling the quantification of $3 \alpha$-ketocorticosteroid, $17 \alpha$-estradiol, $17 \beta$-estradiol and hydroxysteroids in amounts as low as 15 fmol. ${ }^{89}$ Using bis(2carbo-pentyloxy-3,5,6-trichlorophenyl) oxalate in 2-octanone as the PO-CL substrate, it was possible to determine butyl rhodamine $B$ with detection limits of $n \mathrm{~mol} \mathrm{~L}^{-1}$, on a microfluidic chip with liquid-liquid extraction. ${ }^{90}$ The analysis of the pesticide $N$-methylcarbamate in environmental samples by PO-CL was achieved using a flow injection system with sodium dodecyl sulfate micellar media, decreasing the hydrolysis rate of the oxalic ester substrate. The detection limits obtained were about $10 \mathrm{ng} \mathrm{mL}^{-1}$, which, according to the authors, are comparable to that reported for other methods. ${ }^{91}$

The peroxyoxalate system has also been used for the determination of phenol and 4methylphenol in urine samples using HPLC post-column derivatization. After chromatographic separation, the phenols were transformed with dansyl chloride into fluorescent derivatives, which were subsequently detected by PO-CL acting as activators in the system. The detection limits achieved by this method were considerably lower than with other methodologies. ${ }^{92}$

The peroxyoxalate system has been utilized to quantify the amount of trans-resveratrol bound to a molecular imprinted polymer, consisting of uniform trans-resveratrol coated microspheres, with poly(vinyl alcohol) as glue. The low detection limit of $0.1 \mu \mathrm{g} \mathrm{mL}{ }^{-1}$, indicates that this system can be used to analyze trans-resveratrol in various samples. ${ }^{93}$ Using a microfluidic device, the antioxidant capacity of some plant-food based antioxidants, like $\beta$ carotene, $\alpha$-tocopherol and quercetin has been determined with the PO-CL. The principle of the assay is based on the destruction of the hydrogen peroxide by the antioxidants, which leads to the inhibition of light emission from the PO reaction. ${ }^{94}$

In the field of environmental analytical chemistry, PO-CL has been applied to measure the concentration of free chlorine in tap water, including the development of a new flow injection analysis system. ${ }^{95}$ The influence of the analysis conditions like solvent, reactants concentration, structure of the activator and the interference by additives on the analytical parameters has also been investigated in order to optimize reaction conditions. ${ }^{95}$ Environmental analysis of polycyclic aromatic hydrocarbons has employed benzo(a)pyrene as fluorescent marker in flow injection cloud-point extraction. The peroxyoxalate system is used here as a tool to evaluate the preconcentration factor, extraction efficiency, sample throughput and analysis time. The authors suggested that this system has great potential for the analysis of PAH's in environmental samples, in a combination with separation techniques, like high performance liquid chromatography and capillary electrophoresis. ${ }^{96}$

A molecular imprinted polymer for chiral recognition was developed, using the peroxyoxalate system as the chemiluminescent detection tool, achieving low detection limits and a short reaction, showing that this system can be useful for fast chiral recognition. The method is 
based on the analysis of the isomers of derivatized dansyl-L-phenylalanine as a template for the synthesis of polymer microspheres which are used as activators in the peroxyoxalate system. ${ }^{97}$

While the analytical application of oxalic acid derivatives, especially substituted esters and amides, has been extensively studied, hardly any information can be found in the literature with respect to free oxalic acid CL, although, Rauhut et al., in one of their early works on the system, concerned mainly with acyl peroxide decomposition reactions, relate also to the reaction of free oxalic acid. ${ }^{98}$ However, it was shown by Albrecht et al. that the reaction of oxalic acid with hydrogen peroxide in acidic media (ethanol/water mixtures, $\mathrm{pH}$ 1), catalyzed by a carbodiimide in the presence of an appropriate activator, ${ }^{\ddagger}$ took place with an intense short-lived chemiluminescence emission with maximum intensity within $0.4 \mathrm{~s}$ after the start of reaction. ${ }^{87,99}$ This reaction is believed to occur with the intermediate formation of monoperoxyoxalate, that might cyclize to the HEI 1,2-dioxetanedione, which can lead to excited state formation by the electron transfer, cleavage and electron back-transfer sequence according to the CIEEL mechanism. ${ }^{100}$ However, at this time, the authors also postulated the possible formation of excited carbon dioxide which might lead to the excitation of the activator by electronic energy transfer (Scheme 12), although this possibility appears improbable nowadays, due to the high energy level of singlet-excited carbon dioxide. ${ }^{70}$

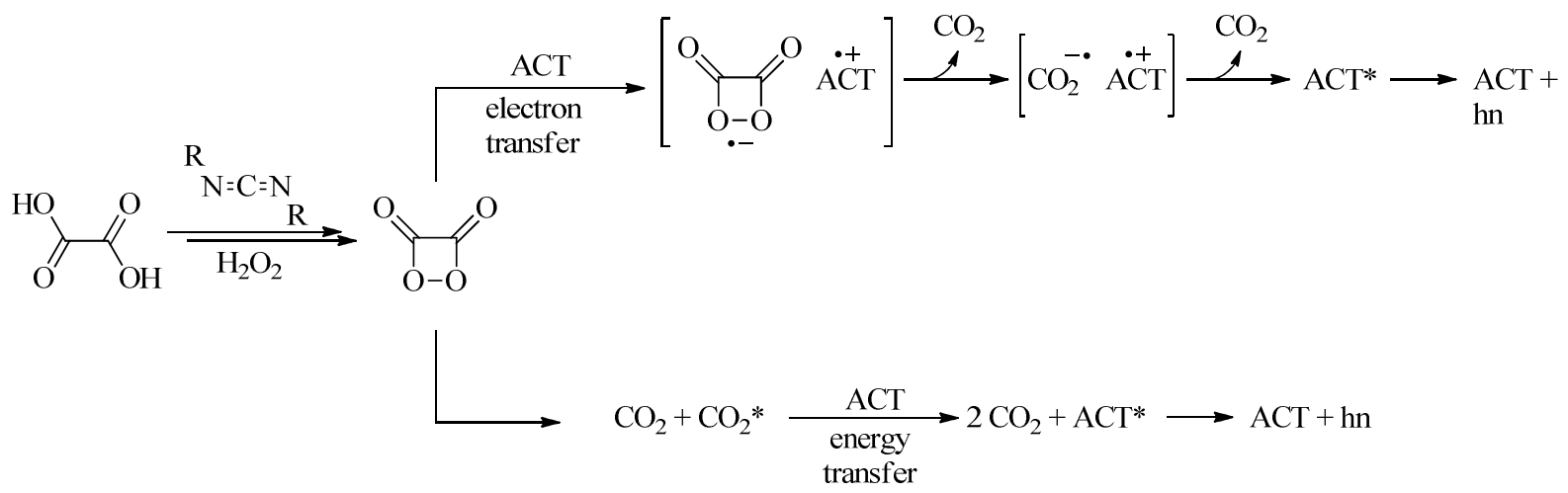

Scheme 12. General chemiexcitation mechanisms proposed for the chemiluminescence reaction of oxalic acid with hydrogen peroxide, catalyzed by a carbodiimide, in the presence of an activator (ACT).

The CL emission obtained under certain reaction conditions using free oxalic acid as reagent has been applied for several biochemical and biomedical applications. The Albrecht research group has developed a sensitive analytical assay for the determination of oxalate in human body fluids and cells, by which it was possible to determine very low concentrations of oxalate (200

$\ddagger$ The compound responsible for CL emission in the system is here called "activator (ACT)", in agreement with the nomenclature utilized throughout this work, it should just be noted that the Albrecht group in their work related to the peroxyoxalate system called this kind of compounds "fluorescer". 
nmol L $\left.{ }^{-1}\right) \cdot{ }^{101,102}$ Using this highly sensitive assay it was shown that the mean serum oxalate level

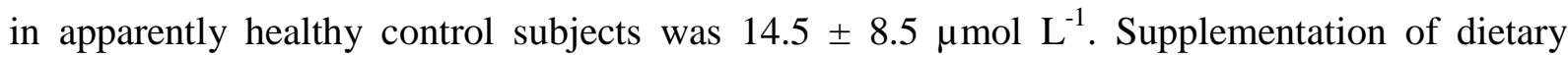
ascorbic acid led to an increase in serum oxalate level. While serum oxalate concentrations of calcium oxalate stone formers $\left(16.4 \pm 9.8 \mu \mathrm{mol} \mathrm{L}{ }^{-1}\right)$ were not significantly different from the control group, an extreme increase in amount of serum oxalate is evident in haemodialysis patients. The serum oxalate concentration decreased during dialysis treatment from $141.4 \pm 32.1$ $\mu \mathrm{mol} \mathrm{L}{ }^{-1}$ to $36.4 \pm 12.7 \mu \mathrm{mol} \mathrm{L}$. The method was also utilized to determine the intracellular oxalate concentrations in erythrocytes, thrombocytes, granulocytes, and monocytes, which was shown to exceed that in the plasma by at least two orders of magnitude, independent of the cell type. ${ }^{101,102}$ Using 9,10-Diphenylanthracene as activator $\left(150 \mathrm{mg} \mathrm{L}^{-1}\right.$ in ethanolic dicyclohexylcarbo-diimide (DCC) solution, $\mathrm{pH} \mathrm{1)}$ and in the presence of $0.1 \mathrm{~mol} \mathrm{~L}^{-1}$ oxalic acid solution, determination of $\mathrm{H}_{2} \mathrm{O}_{2}$ can be achieved down to a limiting concentration of $10^{-8} \mathrm{~mol} \mathrm{~L} \mathrm{~L}^{-1}$, with a maximum emission intensity reached within $0.4 \mathrm{~s}$ and the reaction being completed to $80 \%$ within $2 \mathrm{~s}$ after start. This kinetic characteristic makes this procedure suitable for the determination of enzymes or substrates which produce or destroy $\mathrm{H}_{2} \mathrm{O}_{2}$ (e.g. glucose, oxidases and peroxidases). ${ }^{100}$ The assay system oxalate / DCC / $\mathrm{H}_{2} \mathrm{O}_{2}$ also permits the quantitative analysis of porphyrins in urine down to the concentration of about $250 \mu \mathrm{g} \mathrm{L}^{-1}$, although still containing some problems related to standardization. The use of a suitable photodetector with a maximum sensitivity within a narrow range of the fluorescence (chemiluminescence) maximum for porphyrins (about $630 \mathrm{~nm}$ ) is a basic requirement for sufficiently high sensitivity or further enhancement of sensitivity. ${ }^{100,103}$ Quantification of organo-phosphorous compounds ("Schrader-type") by CL is also possible by generation of highly fluorescent indoxyl (1H-indol-3-ol) and indigo white by reaction of the phosphorous compound with peroxide and indole. The concentration of the indoxyl formed in the assay is determined by the DCC catalyzed oxalic acid reaction with hydrogen peroxide, where indoxyl acts as activator (Scheme 13). ${ }^{104}$ There is a linear dependence of the CL emission intensity of the system oxalic acid / peroxide / carbodiimide and the initial concentration of organo-phosphorous compounds ( $\mathrm{RO}) \mathrm{R} ' \mathrm{P}(\mathrm{O}) \mathrm{X}\left(\mathrm{R}=\right.$ alkyl, $\mathrm{R}^{\prime}$ = alkyl, aryl, alkoxy, dialkylamino, $\mathrm{X}=\mathrm{CN}$, F, etc.) utilized for the formation of the fluorescent indole derivatives. Therefore, this technique can be used as a suitable alternative for the quantification of such compounds at very low concentrations $\left(<1 \mathrm{pmol} \mathrm{L}^{-1}\right)$ that play a role not only as biocides but also as chemical weapons. $^{104}$

Furthermore, a new chemiluminescent substrate for the alkaline phosphatase label used in a Hybritech $^{\circledR}$ immunoassay of prostate-specific antigen (PSA) has been evaluated. Unlike the previously described chemiluminescence immunoassay test for PSA with acridinium derivatives as label, in this assay the substrate utilized was indoxyl phosphate. Enzymatic hydrolysis of this substrate yields indoxyl, an efficient activator in peroxyoxalate chemiluminescence. ${ }^{104,105} \mathrm{~A}$ flash-like kinetic behavior of the emission intensity was observed in this system, which is also characteristic for the direct luminol- or acridinium-labeled luminescence immunoassay. The chemiluminescent system used (oxalic acid / carbodiimide / $\mathrm{H}_{2} \mathrm{O}_{2}$ in ethanol/water mixtures) yields a signal that ranges over three orders of magnitude, depending on the concentration of 
indoxyl released by enzymatic hydrolysis of indoxyl phosphate, indicating that this assay can conveniently be used for converting the colorimetric alkaline phosphatase-based enzyme immunoassays.

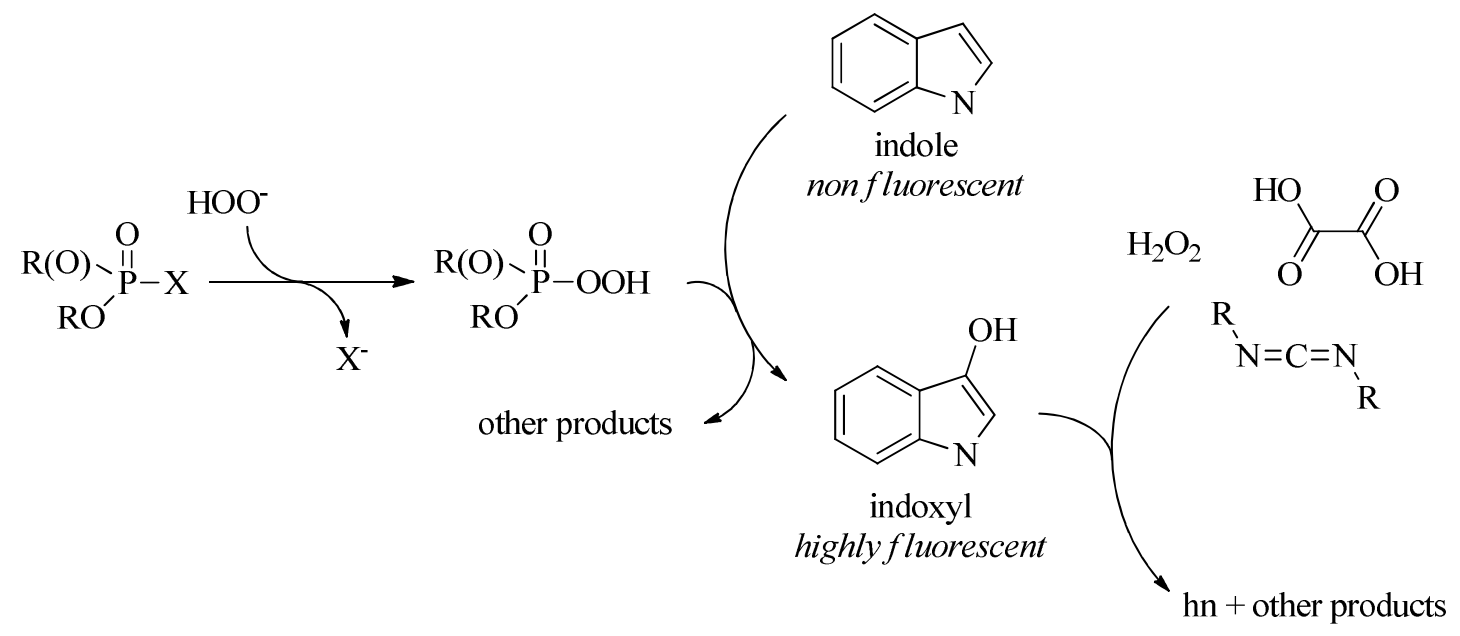

Scheme 13. Reaction scheme for the quantification of organo-phosphorous compounds using peroxyphosphoric acid mediated transformation of indole to indoxyl and its quantification by the oxalic acid / DCC / hydrogen peroxide system.

\section{Conclusions}

In conclusion we hope that we could show the interested reader of this revision the state-of-theart of research on mechanistic aspects of the peroxyoxalate systems and also indicate the principle of analytical applications of the system, using some recent examples. Mechanistic studies during almost 50 years contributed to the understanding of the chemical reaction mechanisms involved in ground-state surface transformations which lead to the formation of a high-energy intermediate. The structure of this high-energy intermediate is still not yet exactly known, although recent studies point to 1,2-dioxetanedione as the most probable candidate for the high-energy intermediate. The interaction of this high-energy intermediate with an appropriate activator, in the chemiexcitation step, where the chemical energy is transformed into electronic excitation energy, appears to involve the CIEEL sequence, as indicated by recent results obtained in our research group. The peroxyoxalate chemiluminescence has been utilized in several analytical applications and in this work we have highlighted the general application principle and provided some interesting recent examples. 


\section{Acknowledgements}

Luiz F. M. L. Ciscato, Felipe A. Augusto, Fernando H. Bartoloni and Wilhelm. J. Baader thank the Fundação de Amparo à Pesquisa do Estado de São Paulo (FAPESP), Deutscher Akademischer Austauschdienst (DAAD) and Coordenadoria de Aperfeiçoamento de Pessoal de Ensino Superior (CAPES) for financial support. Dieter Weiss, Steffen Albrecht, Herbert Brandl and Thomas Zimmermann want to thank Prof. Roland Mayer, former head of the Institut für Organische Chemie of the Technische Universität Dresden, for his constant and unlimited support and promotion of research activities related to applied chemiluminescence.

\section{References}

1. Campbell, A. K. Chemiluminescence: Principles and Applications in Biology and Medicine; Ellis Horwood Ltd.: Chichester, 1988.

2. Wiedemann, E. Ann. Phys. Chem. 1888, 34, 446.

3. Radziszewski, B. Ber. Chem. Ges. 1877, 10, 70.

4. Albrecht, H. O. Z. Phys. Chem. 1928, 136, 321.

5. Gleu, K.; Petsch, W. Angew. Chem. 1935, 48, 57.

6. Adam, W. Pure Appl. Chem. 1980, 52, 2591.

7. Schuster, G. B.; Schmidt, S. P. Adv. Phys. Org. Chem. 1982, 18, 187.

8. Turro, N. J. Modern Molecular Photochemistry; University Science Books: Sausalito, 1991.

9. Bartoloni, F. H.; Ciscato, L. F. M. L.; Peixoto, M. M. M.; Santos, A. P. F.; Santos, C. S.; Oliveira, S.; Augusto, F. A.; Pagano A. P. E.; Baader, W. J. Quim. Nova 2011, 34, 544.

10. (a) Marcus, R. A. J. Chem. Phys. 1965, 43, 2654. (b) Marcus, R. A.; Siders, P. J. Phys. Chem. 1982, 86, 622. (c) Marcus, R. A. In Electron Transfer - From Isolated Molecules to Biomolecules; Jortner, J.; Bixon, M. Eds.; John Wiley \& Sons: Chichester, 1999.

11. Baader, W. J.; Stevani, C. V.; Bastos, E. L. In: The Chemistry of Peroxides; Rappoport, Z. Ed.; John Wiley \& Sons: Chichester, 2006, 2, p 1211.

12. (a) Adam, W.; Baader, W. J. Angew. Chem., Int. Ed. 1984, 23, 166. (b) Adam, W. In The Chemistry of Peroxides; Patai, S. Ed.; John Wiley \& Sons: Chichester, 1983, p 829. (c) Adam. W.; Baader, W. J.; Babatsikos, C.; Schmidt, E. Bull. Soc. Chim. Belg. 1984, 93, 605.

13. Schuster, G. B. Acc. Chem. Res. 1979, 12, 366.

14. (a) Schmidt, S. P.; Schuster, G. B. J. Am. Chem. Soc. 1980, 102, 306. (b) Schmidt, S. P.; Schuster, G. B. J. Am. Chem. Soc. 1978, 100, 1966. (c) Adam, W.; Simpson, A.; Yani, F. J. Phys. Chem. 1974, 78, 2559. (d) Adam, W.; Cueto, O. J. Am. Chem. Soc. 1979, 101, 6511. (e) Turro, N. J.; Chow, M. -F. J. Am. Chem. Soc. 1980, 102, 5058.

15. (a) Koo, J.-Y.; Schuster, G. B. J. Am. Chem. Soc. 1977, 99, 5403. (b) Koo, J. -Y.; Schuster, G. B. J. Am. Chem. Soc. 1978, 100, 4496.

16. Koo, J.-Y.; Schmidt, S. P.; Schuster, G. B. Proc. Natl. Acad. Sci. USA 1978, 75, 30. 
17. Catalani, L.H.; Wilson, T. J. Am. Chem. Soc. 1989, 111, 2633.

18. Wilson, T. Photochem. Photobiol. 1995, 62, 601.

19. (a) Schaap, A. P.; Gagnon, S. D. J. Am. Chem. Soc. 1982, 104, 3504. (b) Schaap, A. P.; Chen, T-S.; Handley, R. S.; De Silva, R.; Giri, B. P. Tetrahedron Lett. 1987, 28, 1155. (c) Schaap, A. P.; Handley, R. S.; Giri, B. P. Tetrahedron Lett. 1987, 28, 935. (d) Schaap, A. P.; Sandison, M. D.; Handley, R. S. Tetrahedron Lett. 1987, 28, 1159.

20. (a) Bronstein, I.; Voyta, J. C.; Lazzari, K. G.; Murphy, O.; Edwards, B.; Kricka, L. J. Biotechniques, 1992, 12, 748. (b) Lim, T.; Komoda, Y.; Nakamura, N.; Matsunaga, T. Anal. Chem. 1999, 71, 1298. (c) Beck, S.; Köster, H. Anal. Chem. 1990, 62, 2258. (d) Adam, W.; Reinhardt, D.; Saha-Möller, C. R. Analyst, 1996, 121, 1527.

21. (a) Nery, A. L. P.; Baader, W. J. Quim. Nova 2001, 24, 626. (b) Nery, A.L.P.; Röpke, S.; Catalani, L. H.; Baader, W.J. Tetrahedron Lett. 1999, 40, 2443. (c) Nery, A.L.P.; Weiss, D.; Catalani, L. H.; Baader, W.J. Tetrahedron 2000, 56, 5317.

22. (a) Watanabe, N.; Kobayashi, H.; Azami, M.; Matsumoto, M. Tetrahedron 1999, 55, 6831. (b) Adam, W.; Reinhardt, D. Liebigs Ann. 1997, 1359. (c) Adam, W.; Bronstein, I.; Trofimov, A. V.; Vasil'ev, R. F. J. Am Chem. Soc. 1999, 121, 958. (d) Adam, W.; Trofimov, A. V. J. Org. Chem. 2000, 65, 6474. (e) Adam, W.; Matsumoto, M.; Trofimov, A.V. J. Am. Chem. Soc. 2000, 122, 8631. (f) Matsumoto, M. Photochem. Photobiol. C: Photochem. Rev. 2004, 5, 27. (g) Tanimura, M.; Watanabe, N.; Ijuin, H. K.; Matsumoto, M. J. Org. Chem. 2010, 75, 3678 .

23. Ciscato, L. F. M. L.; Bartoloni, F. H.; Weiss, D.; Beckert, R.; Baader, W.J. J. Org. Chem. 2010, 75, 6574.

24. Chandross, E. A. Tetrahedron Lett. 1963, 12, 761.

25. Chandross, E. A. In Bioluminescence and Chemiluminescence: Fundamentals and Applied Aspects; Campbell, A. K.; Kricka, L. J.; Stanley, P. E. Eds.; John Wiley: New York, 1994, p 8-15.

26. Stevani, C. V.; Baader, W. J. Quim. Nova 1999, 22, 715.

27. Rauhut, M. M. Acc. Chem. Res. 1969, 2, 80.

28. Rauhut, M. M.; Roberts, B. G.; Semsel, A. M. J. Am. Chem. Soc. 1966, 88, 3604.

29. Bollyky, L. J.; Whitman, R. H.; Roberts, B. G.; Rauhut, M. M. J. Am. Chem. Soc. 1967, 89, 6523.

30. Rauhut, M. M.; Bollyky, L. J.; Roberts, B. G.; Loy, M.; Whitman, R. H.; Iannotta, A. V.; Semsel, A. M.; Clarke, R. A. J. Am. Chem. Soc. 1967, 89, 6515.

31. Maulding, D. R.; Clarke, R. A.; Roberts, B. G.; Rauhut, M. M. J. Org. Chem. 1968, 33, 250.

32. Catherall, C. L. R.; Palmer, T. F. J. Chem. Soc., Faraday Trans. 2 1984, 823.

33. Orosz, G. Tetrahedron 1989, 45, 3493.

34. Alvarez, F.; Parekh, N. J.; Matuszewski, B.; Givens, R. S.; Higuchi, T.; Schowen, R. L. J. Am. Chem. Soc. 1986, 108, 6435.

35. Orlovic, M.; Schowen, R. L.; Givens, R. S.; Alvarez, F.; Matuszewski, B.; Parekh, N. J. Org. Chem. 1989, 54, 3606. 
36. Hanaoka, N.; Givens, R. S.; Schowen, R. L.; Kuwana, T. Anal. Chem. 1988, 60, 2193.

37. Stevani, C. V.; Lima, D. F.; Toscano, V. G.; Baader, W. J. J. Chem. Soc., Perkin Trans. 2 1996, 989.

38. Milofsky, R. E.; Birks, J. W. Anal. Chem. 1990, 62, 1050.

39. Milofsky, R. E.; Birks, J. W. J. Am. Chem. Soc. 1991, 113, 9715.

40. Neuvonen, H. J. Chem. Soc., Perkin Trans. 2 1995, 945.

41. Neuvonen, H. J. Chem. Soc., Perkin Trans. 2 1994, 89.

42. (a) Kirsch, J. F.; Jencks, W. P. J. Am. Chem. Soc. 1964, 86, 837. (b) Kirsch, J. F.; Jencks, W. P. J. Am. Chem. Soc. 1964, 86, 833.

43. Neuvonen, H. J. Biolumin. Chemilumin. 1997, 12, 241.

44. Hadd, A. G.; Birks, J. W. J. Org. Chem. 1996, 61, 2657.

45. Hadd, A. G.; Robinson, A. L.; Rowlen, K. L.; Birks, J. W. J. Org. Chem. 1998, 63, 3023.

46. Hadd, A. G.; Seeber, A.; Birks, J. W. J. Org. Chem. 2000, 65, 2675.

47. Da Silva, S. M.; Casallanovo, F.; Oyamaguchi, K. H.; Ciscato, L. F. M. L.; Stevani, C. V.; Baader, W. J. Luminescence 2002, 17, 313.

48. Chokshi, H. P.; Barbush, M.; Carlson, R. G.; Givens, R. S.; Kuwana, T.; Schowen, R. L. Biomed. Chromatog. 1990, 4, 96.

49. Hohman, J. R.; Givens, R. S.; Carlson, R. G.; Orosz, G. Tetrahedron Lett. 1996, 37, 8273.

50. Stevani, C. V.; Campos, I. P. A.; Baader, W. J. J. Chem. Soc., Perkin Trans. 2 1996, 1645.

51. Stevani, C. V.; Baader, W. J. J. Phys. Org. Chem. 1997, 10, 593.

52. Da Silva, S. M. Ph. D. Thesis, University of São Paulo, 2004.

53. Lang, A. P. Master Thesis, University of São Paulo, 2008.

54. Bollyky, L. J.; Loy, M.; Roberts, B. G.; Whitman, R. H.; Iannotta, A. V.; Semsel, A. M.; Clarke, R. A. $153^{\text {rd }}$ National Meeting of the American Chemical Society, Miami Beach, Florida, USA, April, 1967: Abstract O-169.

55. Cordes, H. F.; Richter, H. P.; Heller, C. A. J. Am. Chem. Soc. 1969, 91, 7209.

56. De Corpo, J. J.; Baronavski, A.; McDowell, M. V.; Saalfeld, F. E. J. Am. Chem. Soc. 1972, 94, 2880.

57. Orosz, G.; Givens, R. S.; Schowen, R. L. Anal. Chim. Acta 1992, 266, 219.

58. (a) Sigvardson, K. W.; Birks, J. W. Anal. Chem. 1983, 55, 432. (b) Sigvardson, K. W.; ennish, J. M.; Birks, J. W. Anal. Chem. 1984, 56, 1096.

59. Weinberger, R.; Mannan, C. A.; Cerchio, M. J. Chromatogr. 1984, 288, 445.

60. Mann, B.; Grayeski, M. L. Anal. Chem. 1990, 62, 1532.

61. Stauff, J.; Jaeschke, W.; Schlogl, G. Z. Z. Phys. Chem. 1976, 99, 37.

62. Lee, J. H.; Rock, J. C.; Park, S. B.; Schlautman, M. A.; Carraway, E. R. J. Chem. Soc., Perkin Trans. 2 2002, 802.

63. Lee, J. H.; Rock, J. C.; Schlautman, M. A.; Carraway, E. R. J. Chem. Soc., Perkin Trans. 2 2002, 1653.

64. Lee, J. H.; Je, J.; Schlautman, M. A.; Carraway, E. R. Chem. Commun. 2003, 270.

65. Stevani, C. V.; Baader, W. J. J. Chem. Res. 2002, 430. 
66. Baumstark, A. L.; Landis, M. E.; Brooks, P. J. Org. Chem. 1979, 44, 4251.

67. Bos, R.; Barnett, N. W.; Dyson, G. A.; Lim. K. F.; Russel, R. A.; Watson, S. P. Anal. Chim. Acta 2003, 502, 141.

68. Tonkin, S. A.; Bos, R.; Dyson, G. A.; Lim, K. F.; Russel, R. A.; Watson, S. P.; Hindson, C. M.; Barnett, N. W. Anal. Chim. Acta 2008, 614, 173.

69. Dufraisse, C.; Velluz, L. C. R. Chim. 1939, 209, 516.

70. Lechtken, P.; Turro, N. J. Mol. Photochem. 1974, 6, 95.

71. (a) Richardson, W. H.; Hodge, V. F. J. Am. Chem. Soc. 1971, 93, 3996. (b) Adam, W.; Baader, W. J. J. Am. Chem. Soc. 1985, 107, 410.

72. Catherall, C. L. R.; Palmer, T. F. J. Chem. Soc., Faraday Trans. 2 1984, 837.

73. Stevani, C. V.; Da Silva, S. M.; Baader, W. J. Eur. J. Org. Chem. 2000, 4037.

74. Silva, S. M.; Wagner, K.; Weiss, D.; Beckert, R.; Baader, W. J. Luminescence 2002, 17, 362.

75. Ciscato, L. F. M. L.; Bartoloni, F. H.; Bastos, E. L.; Baader, W. J. J. Org. Chem. 2009, 74, 8974.

76. Bartoloni, F. H.; Ciscato, L. F. M. L.; Augusto, F. A.; Baader, W. J. Quim. Nova 2010, 33, 2055.

77. Garcia-Campana, A. M.; Baeyens, W. R. G.; Cuadros-Rodriguez, L.; Barrero, F. A.; Bosque-Sendra, J. M.; Gamiz-Gracia, L.; Curr. Org. Chem. 2002, 6, 1.

78. Gamiz-Gracia, L.; Garcia-Campana, A. M.; Huertas-Perez, J. F.; Lara, F. J. Anal. Chim. Acta 2009, 640, 7.

79. Garcia-Campana, A. M.; Lara, F. J.; Gamiz-Gracia, L.; Huertas-Perez, J. F. TRAC - Trend Anal. Chem. 2009, 28, 973.

80. Tsunoda, M.; Imai, K. Anal. Chim. Acta 2005, 541, 13.

81. Lin, J. M.; Yamada, M. TRAC - Trend Anal. Chem. 2003, 22, 99.

82. (a) Wada, M.; Inoue, K.; Ihara, A.; Kishikawa, N.; Nakashima, K.; Kuroda, N. J. Chromatogr., A 2003, 987, 189. (b) Garcia-Campana, A. M.; Gamiz-Gracia, L.; Baeyens, W. R. G.; Barrero, F. A. J. Chromatogr. B: Anal. Technol. Biomed. Life Sci. 2003, 793, 49. (c) Appelblad, P.; Jonsson, T.; Backstrom, T.; Irgum, K. Anal. Chem. 1998, 70, 5002. (d) Carr, A.; Dickson, J.; Dickson, M.; Milofsky, R. Chromatographia 2002, 55, 687.

83. (a) Sun, S.; Tang, Y.; Fu, Q.; Chen, J.; Zhang, G. Anal. Lett. 2009, 42, 1868. (b) Lee, J. H.; Je, J.; Tartaglia, A.; Hur, J.; Schlautman, M. A.; Carraway, E. R. J. Photochem. Photobiol. A 2006, 182, 28.

84. (a) Nakamura, M. M.; Saraiva, S. A.; Coichev, N. Anal. Lett. 2000, 33, 391. (b) Steijger, O. M.; Rodenburg, P. H. M.; Lingeman, H.; Brinkman, U. A. T.; Holthuis, J. J. M. Anal. Chim. Acta 1992, 266, 233.

85. (a) Williams, D. C.; Huff, G. F.; Seitz, W. R. Anal. Chem. 1976, 48, 1003. (b) Scott, G.; Seitz, W. R.; Ambrose, J. Anal. Chim. Acta 1980, 115, 221. (c) Vanzoonen, P.; Kamminga, D. A.; Gooijer, C.; Velthorst, N. H.; Frei, R. W. Anal. Chim. Acta 1985, 167, 249. (d) Poulsen, J. R.; Birks, J. W.; Gubitz, G.; Vanzoonen, P.; Gooijer, C.; Velthorst, N. H.; Frei, 
R. W. J. Chromatogr. 1986, 360, 371. (e) Nozaki, O.; Iwaeda, T.; Kato, Y. J. Biolumin. Chemilumin. 1995, 10, 339.

86. (a) Nakashima, K.; Kuroda, N.; Kawaguchi, S.; Wada, M.; Akiyama, S. J. Biolum. Chemilum. 1995, 10, 185. (b) Grayeski, M. L., Woolf, E. J., Helly, P. J. Anal. Chim. Acta 1986, 183, 207.

87. Albrecht, S.; Beckert, R.; Böhm, W.-D. J. Clin. Chem. Clin. Biochem. 1989, 27, 451.

88. Arnous, A.; Petrakis, C.; Makris, D. P.; Kefalas, P. J. Pharm. and Toxic. Met. 2002, 48, 171.

89. Appelblad, P.; Irgum, K. J. Chromatogr. A 2002, 995, 151.

90. Shen, H.; Fang, Q.; Fang, Z.-L. Lab Chip 2006, 6, 1387.

91. Soto-Chinchilla, J. J.; Garcia-Campana, A. M.; Gamiz-Gracia, L.; Cuadros-Rodriguez, L.; Vidal, J. L. M. Anal. Chim. Acta 2004, 524, 235.

92. Orejuela, E.; Silva, M. Analyst 2002, 127, 1433.

93. Wang, L.; Zhang, Z.-J. Anal. Chim. Acta 2007, 592, 115.

94. Amatatongchai, M.; Hofmann, O.; Nacapricha, D.; Chailapakul, O.; deMello, A. J. Anal. Bioanal. Chem. 2007, 387, 277.

95. Nakamura, M. M.; Coichev, N.; Lin, J. M.; Yamada, M. Anal. Chim. Acta 2003, 484, 101.

96. Song, G. Q.; Lu, C.; Hayakawa, K.; Lin, J. M. Anal. Bioanal. Chem. 2006, 384, 1007.

97. Wang, L.; Zhang, Z.-J.; Huang, L.-G. Anal. Bioanal. Chem. 2008, 390, 1431.

98. Rauhut, M. M.; Sheehan, D.; Clarke, R. A.; Semsel, A. M. Photochem. Photobiol. 1965, 4, 1097.

99. Albrecht, S.; Brandl, H.; Böhm W.-D.; Beckert, R.; Kroschwitz, H.; Neumeister, V. Anal. Chim. Acta 1991, 255, 413.

100.Albrecht, S.; Brandl, H.; Adam, W. Nachr. Chem. Tech. Lab. 1992, 40, 547.

101.Albrecht, S.; Hornak, H.; Freidt, T.; Böhm, W.-D.; Weiss, K.; Reinschke, A. J. Biolumin. Chemilumin. 1993, 8, 21.

102.(a) Albrecht, S.; Brandl, H.; Schönfels, C. Angew. Chem. Int. Ed. Engl. 1994, 33, 1780. (b) Albrecht, S.; Brandl, H.; Schönfels, C. Angew. Chem. 1994, 106, 1864.

103.Albrecht, S.; Brandl, H.; Koestler, E. Z. Klin. Med. 1989, 44, 2071.

104.(a) Albrecht, S.; Brandl, H.; Böhm, W.-D. Fresenius J. Anal. Chem. 1990, 337, 93. (b) Albrecht, S.; Brandl, H.; Böhm, W.-D. Fresenius J. Anal. Chem. 1992, 343, 176.

105.Albrecht, S.; Brandl, H.; Freidt, T. Clin. Chem. 1994, 40, 1970. 


\section{Authors' Biographies}

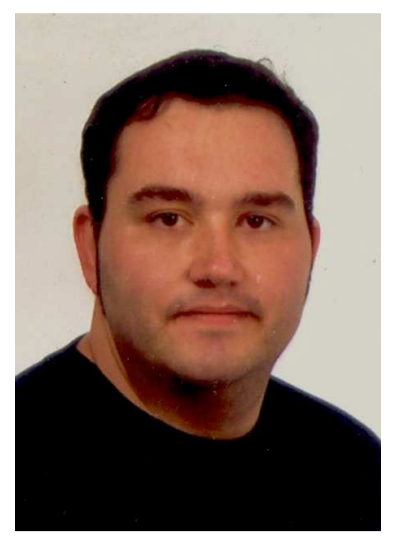

Luiz Francisco Monteiro Leite Ciscato was born in 1979 in São Paulo (Brazil), and studied Chemistry at the University of São Paulo. Between 2003 and 2008 he worked on his Ph.D. degree under the supervision of Prof. W. J. Baader at the University of São Paulo. During this time, in 2005, he stayed for one year in the laboratory of Prof. Rainer Beckert at the Friedrich Schiller-University of Jena, Germany. Thereafter, he worked as a postdoc associate from 2008 to 2009 again under the supervision of Prof. Rainer Beckert. From 2010 to 2011 he worked on his second postdoctoral project at the University of São Paulo, developing new chemiluminescent probes for the detection of reactive oxygen species in biological media. He currently has a position as Professor at the Federal University of the ABC (UFABC), in Santo André, Brazil.

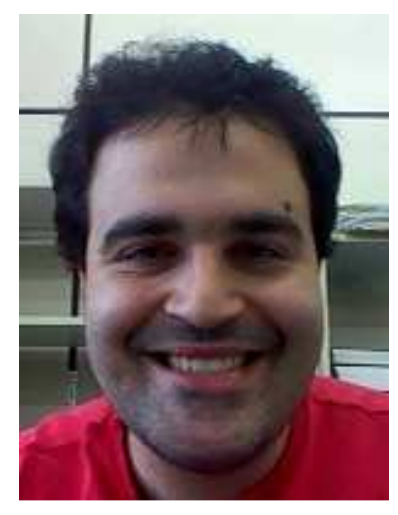

Felipe Alberto Augusto was born in 1988 in São Paulo (Brazil) and studied Chemistry at the University of São Paulo, where he got his Bachelor's degree in 2010. He is presently a graduate student in the laboratory of Prof. W. J. Baader at the same university and works with peroxyoxalate chemiluminescence and computational chemistry. 


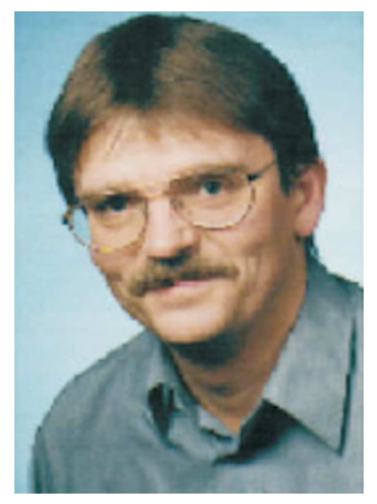

Dieter Weiss was born in 1958, studied Chemistry at the University of Jena, Germany and got his Diplom and Ph.D. degrees at the same university, working on conducting polymers. In 1999, he had a short stay in the laboratory of Prof. W. J. Baader at the University of São Paulo, Brazil. In 2000, he accomplished his Habilitation about fluorescent steroid derivatives and since 2003 he is a Privat Dozent at the University of Jena, where he works with the synthesis of fluorescent substances, fluorescent ligands and organic chemiluminescence reactions.

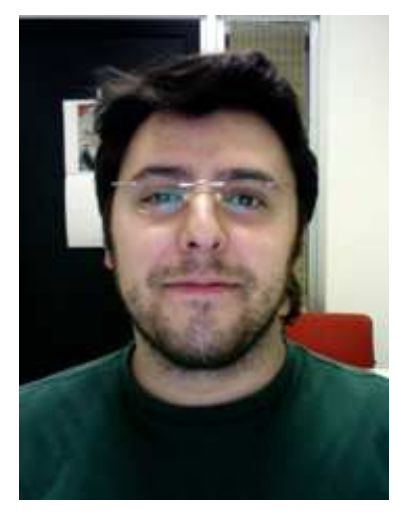

Fernando Heering Bartoloni was born in São Caetano do Sul (Brazil) in 1984, and studied Chemistry at the University of São Paulo. In 2006 he started his Ph.D. investigations about the utilization of the peroxyoxalate reaction in aqueous medium and the synthesis and studies of new alpha-peroxylactone derivatives, under the supervision of Prof. W. J. Baader, obtaining his Ph.D. degree in 2011. He has since been working as postdoc associate on the isolation and photophysical and photochemical characterization of highly fluorescent plant metabolites at the Federal University of the ABC (UFABC), Brazil, under the supervision of Prof. Erick L. Bastos. 


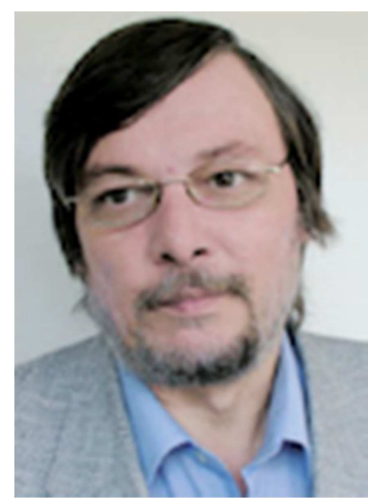

Steffen Albrecht was born in 1957 and studied Chemistry at the Technical University of Dresden (Germany) and got his Ph.D. degree at the same university in 1985, working in phosphororganic synthetic chemistry, under the supervision of Prof. E. Herrmann. Since 1985 he is the responsible Scientist in the Gynecological Clinical Laboratory at the Technical University of Dresden, where he obtained his Habilitation in 2008, working on various aspects of applied chemiluminescence. In 2008 he became an Associated Professor at the same University, developing studies on the application of chemiluminescence and bioluminescence reactions, as well as detection methods for several pathological states.

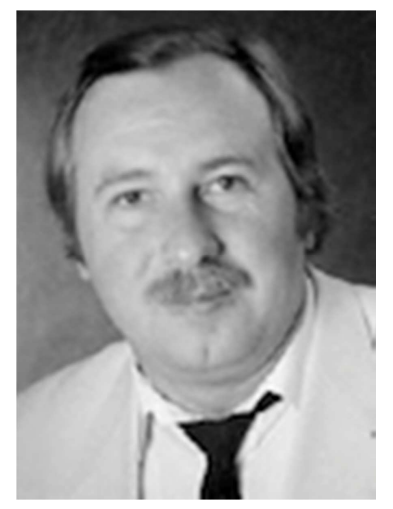

Herbert Brandl was born in 1947 and got his degrees in Chemistry and Biology at the Universities of Erlangen and Munich. Since 1980 he is working as a high-school teacher (Studienrat) for Chemistry and Biology at the Gymnasium of Kaltenkirchen (Germany). His research interests involve the application of chemi-, bio- and triboluminescence, mainly for teaching purpose, as well as oscillating reactions. 


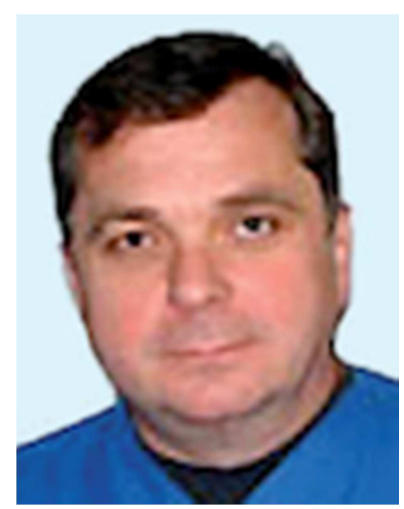

Thomas Zimmermann, born in 1959, studied Medicine between 1978 and 1984 in Szeged (Hungary). From 1985 to 2010 he worked at the Medical Faculty of the Technical University of Dresden (Germany), where he obtained his Habilitation in 2008. In 2010 he was also named Oberarzt at the Hospital of Freiberg (Germany). His research interest involves applied chemiluminescence, RNA and DNA analysis during surgery, free radicals and reactive $\mathrm{C} 1$ and $\mathrm{C} 2$ building blocks in oncological and inflammation processes.

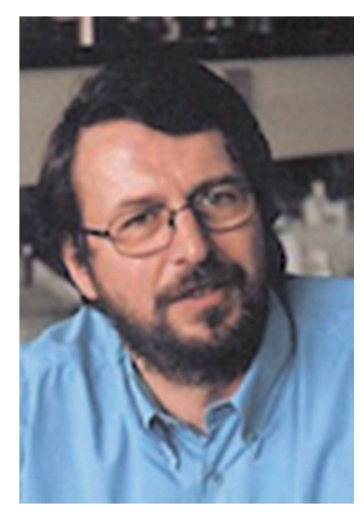

Wilhelm Josef Baader was born in 1954 in Spalt (Germany), and studied Chemistry at the University of Würzburg, where he got his Ph.D. in 1980 under the supervision of Prof. W. Adam, working on the mechanism of 1,2-dioxetane decomposition. After postdoc studies at the Universities of São Paulo and Konstanz, he became a Professor at the University of São Paulo in 1989, and since then he has been working in the field of mechanistic and applied organic chemiluminescence. 